\title{
The critical effects of matrices on cultured carcinoma cells : Human tumor-derived matrix promotes cell invasive properties
}

\section{Wahbi, Wafa}

2020-04-01

Wahbi , W , Naakka , E , Tuomainen , K, Suleymanova , I , Arpalahti , A, Miinalainen , I, Vaananen, J , Grenman, R , Monni , O , Al-Samadi , A \& Salo , T 2020 , ' The critical effects of matrices on cultured carcinoma cells : Human tumor-derived matrix promotes cell invasive properties ' , Experimental Cell Research , vol. 389 , no. 1 , 111885 . https://doi.org/10.1016/j.yexcr.2020.11

http://hdl.handle.net/10138/325579

https://doi.org/10.1016/j.yexcr.2020.111885

cc_by_nc_nd

acceptedVersion

Downloaded from Helda, University of Helsinki institutional repository.

This is an electronic reprint of the original article.

This reprint may differ from the original in pagination and typographic detail.

Please cite the original version. 

promotes cell invasive properties

3 Wafa Wahbi ${ }_{1,2}$, Erika Naakka ${ }^{1,2 \sharp}$, Katja Tuomainen ${ }^{1,2 \sharp}$, Ilida Suleymanova ${ }^{1,2}$, Annamari Arpalahti ${ }_{1,2}$,

4 Ilkka Miinalainen ${ }^{3}$, Juho Vaananen ${ }^{4}$, Reidar Grenman', Outi Monni ${ }^{4}$, Ahmed Al-Samadi ${ }^{2}{ }^{*}$, , Tuula

5 Salo $1,2,6,788$

61 Department of Oral and Maxillofacial Diseases, Clinicum, Faculty of Medicine, University of

7 Helsinki, Helsinki, Finland

82 Translational Immunology Program, Faculty of Medicine, University of Helsinki

93 Biocenter Oulu Electron Microscopy Core Facility, University of Oulu, Oulu, Finland.

104 Applied Tumor Genomics Research Program, Faculty of Medicine, University of Helsinki, Helsinki,

11 Finland 5 Department of Otolaryngology, Turku University, Turku, Finland.

126 Cancer and Translational Medicine Research Unit, University of Oulu, Oulu, Finland

137 Medical Research Centre, Oulu University Hospital, Oulu, Finland

148 Helsinki University Hospital, Helsinki, Finland

15 \# Designates an equal contribution to this work.

16 \& Supervised the work equally.

17

18

19

20

21

22

23

24

25 Declaration of interests: The authors declare that they have no conflict of interest.

* Corresponding author: Ahmed Al-Samadi, Department of Oral and Maxillofacial Diseases, Clinicum, Biomedicum Helsinki 1, C223b P.O. Box 63 (Haartmaninkatu 8), 00014 University of Helsinki, Helsinki, Finland; E-mail: ahmed.al-samadi@helsinki.fi ; Tel: +358458947224. 


\section{Abstract}

28 The interaction between squamous cell carcinoma (SCC) cells and the tumor microenvironment 29 (TME) plays a major role in cancer progression. Therefore, understanding the TME is essential for 30 the development of cancer therapies.

31 We used four (primary and metastatic) head and neck (HN) SCC cell lines and cultured them on top

32 33 34
8 0 1 2

of or within 5 matrices (mouse sarcoma-derived_Matrigel ${ }^{\circledR}$, rat collagen, human leiomyoma-derived Myogel, human fibronectin, and human fibrin). We performed several assays to study the effects of these matrices on the HNSCC behavior, such as proliferation, migration, and invasion, as well as cell morphology, and molecular gene profile.

Carcinoma cells exhibited different growth patterns depending on the matrix. While fibrin enhanced the proliferation of all the cell lines, collagen did not. The effects of the matrices on cancer cell migration were cell line dependent. Carcinoma cells in Myogel-collagen invaded faster in scratch wound invasion assay. On the other hand, in the spheroid invasion assay, three out of four cell lines invaded faster in Myogel-fibrin. These matrices significantly affected hundreds of genes and a number of pathways, but the effects were cell line dependent.

The matrix type played a major role in HNSCC cell phenotype. The effects of the ECMs were either constant, or cell line dependent. Based on these results, we suggest to select the most suitable matrix, which provides the closest condition to the in vivo TME, in order to get reliable results in in vitro experiments.

Keywords: Cancer, Extracellular Matrix, Invasion, Migration, Tumor Microenvironment. 
Squamous cell carcinoma cells are notably affected by their microenvironment that mainly includes extracellular matrix (ECM) and tumor stromal cells, such as cancer-associated fibroblasts (CAF), immune and endothelial cells (1). ECM is a major component of the TME and it is composed of a variety of proteins, proteoglycans, and polysaccharides (2). The structure and physical properties of tumor-associated ECM differ from normal tissue stroma (3). Changes in the ECM properties may cause variation in collagen deposition, promote the ECM stiffness, and upgrade cell survival and proliferation_(4,5). ECM could also affect tumor stroma cells, such as CAFs, immune and endothelial cells (6). Therefore, understanding the SCC microenvironment is essential for the development of cancer therapies, which targets not only the cancer cells but also their environment that allows them to proliferate and spread. In vitro, cancer cells are generally studied in 2D plastic wells. This usually leads to a loss of several important elements, which could affect the cell behavior and phenotype, making the 2D system not representative of the in vivo situation. In order to provide a more physiological environment for the cells, culture systems using different ECM mimicking three-dimensional matrices were introduced. Even though several matrices, which are extracted from different species such as mouse, rat, bovine, or prepared from non-animal material (7), were proposed to be used in 3D cell culture assays, selecting the most appropriate matrix for each cell type is not straightforward. In spite of presence of several matrices from different origins, a human tumour-derived matrix is still missing from the market. Our group has invented the first tumour-derived matrix "Myogel" which is derived from leiomyoma tissue (8). Myogel has been used in several cancer in vitro studies (1,9-12). Myogel proteome differs greatly from the commonly used mouse sarcomaderived Matrigel (8). We have shown recently that Myogel enhance the proliferation of freshly isolated cancer cells from primary tumor compared to plastic and Matrigel (10). Additionally, based on our recent publication, Myogel also improved the predictability of head and neck cancer drug testing (12). This setup, applying Myogel coated wells in drug testing, could reduce the number of failure clinical trials and reduce the cost of the anti-cancer drug development.

Here, we aimed to investigate the effects of several human- and animal-extracted ECMs, on the head and neck (HN) SCC cells. We used mouse tumor-derived Matrigel ${ }^{\circledR}$, rat tail collagen, human plasma fibronectin, human-derived fibrin, and human tumor-derived Myogel ${ }_{L}$ Bovine serum albumin (BSA) and uncoated wells were used as negative 
controls. We selected four HNSCC cell lines as a model of SCC cells: UT-SCC-24 (tongue) and UTSCC-42 (larynx), including primary (A) and corresponding metastasis (B). We compared the effects of these matrices with the non-coated plastic analyzing cell morphology, proliferation, migration, and invasion. We also studied the effects of these matrices on the molecular profile of these cells using transcriptome profiling.

\section{Materials and Methods}

Cell lines

UT-SCC cell line series, UT-SCC-24A (Primary tongue cancer, RRID:CVCL_7826), UT-SCC-24B (Metastatic tongue cancer, RRID:CVCL_7827) and UT-SCC-42 (larynx), including primary (A, RRID:CVCL_7847) and metastatic (B, RRID:CVCL_7848). Were kindly provided by Prof. Grenman (Department of Otolaryngology, Turku University, Turku, Finland). UT-SCC cells were grown in DMEM-F12 medium (Gibco ${ }^{\text {TM }} /$ Invitrogen, Tokyo, Japan) supplemented with $10 \%$ foetal bovine serum, $1 \%$ penicillin/streptomycin and $250 \mu \mathrm{g} / \mathrm{mL}$ amphotericin B (all from Sigma-Aldrich, St. Louis, Mo, USA). All the cell lines were cultured in a humidified incubator $\left(37^{\circ} \mathrm{C}, 5 \% \mathrm{CO} 2,95 \%\right.$ humidity, Binder, Tuttlingen, Germany).

Locally established four cell lines were isolated from two HNSCC patients, having both primary and metastatic tumors. Details of the cell lines are provided in supplementary Table 1.

Preparation of the wells and light microscope imaging of cells morphology Ninety-six-well plates with black well walls and clear bottoms (Essen Bioscience, Ann Arbor, MI, USA) were used for coating. The plate was placed on ice and $50 \mu \mathrm{L} /$ well of $0.5 \mathrm{mg} / \mathrm{mL}$ Matrigel and collagen (Corning, Corning, NY, USA) were dispensed using cold pipet tips. The plate was placed in the incubator for 30 minutes, then $50 \mu \mathrm{L} /$ well of $0.01 \mathrm{mg} / \mathrm{mL}$ BSA (Sigma-Aldrich), $0.01 \mathrm{mg} / \mathrm{mL}$ fibronectin (Sigma-Aldrich) ${ }_{2} \div 0.5 \mathrm{mg} / \mathrm{mL}$ Myogel (Lab made, see below): 


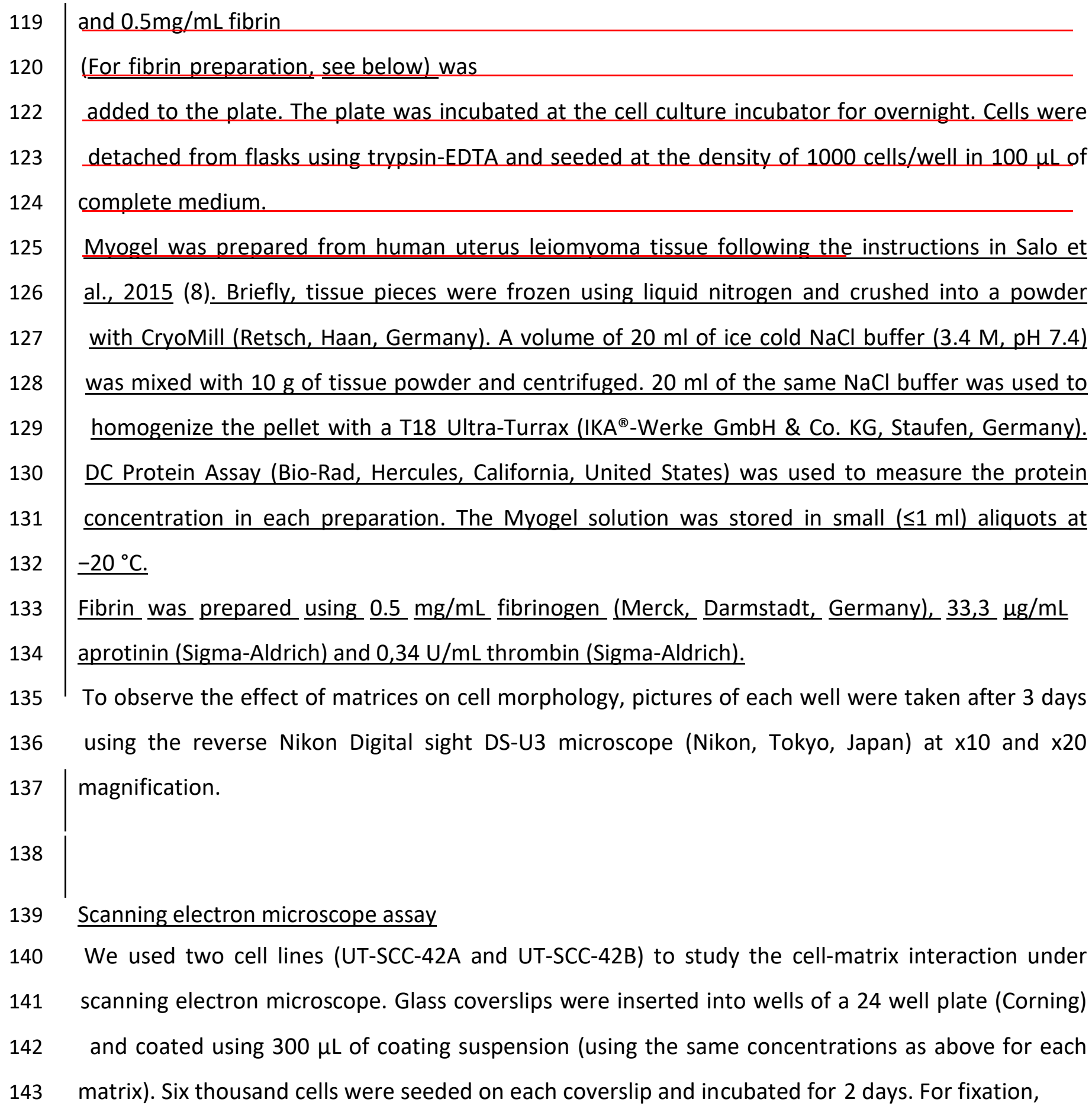


we performed several washing steps with $500 \mu \mathrm{L}$ of phosphate-buffered saline (PBS), and then we added $500 \mu \mathrm{L}$ of $4 \%$ formaldehyde and kept it for 20 minutes at room temperature. After that, we washed the wells with PBS again for 3 times, 5 minutes each. Samples were dehydrated using graded ethanol series and dried using K850 critical point dryer (Quorum Technologies, UK). After drying, samples were attached to aluminium stubs with double-sided carbon tape and coated with $5 \mathrm{~nm}$ of platinum using Q150T ES sputter coater (Quorum Technologies, UK). Samples were imaged with Sigma HD-VP field-emission scanning electron microscope (Carl Zeiss, Oberkochen, Germany).

\section{Proliferation luminescent cell viability assay}

For proliferation, we used the same experiment settings as in the imaging assay. After 3 days, the plate was taken out from the incubator to room temperature for $15 \mathrm{~min}$ before starting the assay. $100 \mu \mathrm{L}$ of CellTiter-Glo was dispensed in each well. The plate was put on a plate shaker (Heidolph, Schwabach, Germany) for $5 \mathrm{~min}$ at $450 \mathrm{rpm}$ and then in plate spinner (Thermo Scientific, Massachusetts, USA) for $5 \mathrm{~min}$ at $1000 \mathrm{rpm}$. Finally, the plate was placed in the BMG PHERAstar FS (BMG Labtech, Offenburg, Germany) plate reader to detect cell viability.

\section{$\underline{\text { Scratch wound cell migration assay }}$}

The same coating protocol was used as before, except that the gels were sucked out before seeding the cells. We seeded the cells at the following density: 25000/well for UT-SCC-24A and UTSCC-42B and 30000/well for UT-SCC-24B and UT-SCC-42A. Matrigel was not used in this experiment as the cells were forming clusters on top of the Matrigel leading to difficulties in getting a smooth scratch. The wound maker (Essen Bioscience) was used to achieve homogeneous scratch wounds.

Wounds were checked under the light microscope and the media was changed for all the wells. The plate was placed in IncuCyte ZOOM incubator (Essen Bioscience), and wounds confluences were monitored using the IncuCyte Live-Cell Imaging System (Essen Bioscience). Images were taken each hour for 20 hours. Supplementary Video (Online Resource 1) shows the migration of the UT-SCC-42A cells on top of Myogel. 


\section{Scratch wound cell invasion assay}

Four gels were used in this experiment: collagen, Myogel-collagen, fibrin, and Myogel-fibrin at the concentration of $1 \mathrm{mg} / \mathrm{mL}$ for all of them, as instructed by the manufacturer (Essen Bioscience). Similar to the migration experiment, wells were coated, cells were seeded and a scratch wound was made. After that, $50 \mu \mathrm{L}$ of the gels were added. Once the gels solidified, $50 \mu \mathrm{L}$ of media was added. The plate was placed in IncuCyte ZOOM incubator, and wounds confluences were monitored using the IncuCyte Live-Cell Imaging System (Essen Bioscience). Images were taken each hour for 48 hours. Supplementary Video (Online Resource 2) shows the invasion of the UTSCC-42A within Myogel-collagen.

\section{Spheroid Invasion Assay}

Cells were seeded at the concentration of 1000 cells/well in $50 \mu \mathrm{L}$ using ultra-low attachment 96well round bottom plate wells (Corning). The plate was incubated for 4 days to allow spheroid formation. Spheroids were embedded in $100 \mu \mathrm{L}$ of Matrigel $(0.5 \mathrm{mg} / \mathrm{mL})$, collagen $(0.5 \mathrm{mg} / \mathrm{mL})$, Myogel-collagen $(0.5 \mathrm{mg} / \mathrm{mL})$, fibrin $(0.5 \mathrm{mg} / \mathrm{mL}$ fibrinogen $+0.3 \mathrm{U} / \mathrm{mL}$ Thrombin $+3.33 \mathrm{mg} / \mathrm{mL}$ Aprotinin), and Myogel-fibrin $(0.5 \mathrm{mg} / \mathrm{mL})$. Gels were allowed to solidify for 30 minutes and then $100 \mu \mathrm{L}$ of DMEM was added into each well. The plate was incubated for 4 days and pictures were taken every day using Nikon Digital sight DS-U3 microscope (Nikon) at x4 magnification. The used protocol is explained in detail in Naakka et al., 2019 (9)-. We analyzed the area covered by cells using ilastik and ImageJ (Wayne Rasband, National Institute of Mental Health, Bethesda, MD, USA). Once ilastik detected area covered by cells, we used a custom plugin, written for ImageJ, to measure the area. The plugin converts the image to black and white image. All pixels outside the area are set to zero, the cells area is set to one. The total area is measured as a number of pixels equal to one.

\section{Microarray}

UT-SCC-24A and UT-SCC-24B cell lines were used to study the effects of different matrices on the molecular gene profile using RNA sequencing transcriptome profiles. Wells of 24 well-plates were coated with $150 \mu \mathrm{L}$ of gels (using the same concentrations as in the imaging assay) and seeded with 150000 cells. Cells were left on the gels for 24 hours and lysed using RLT buffer. RNA was 

extracted using RNeasy Kit (Qiagen, Düsseldorf, Germany) according to manufacturer instructions. In case some clots or fragments of gels existed in the cell lysate, sonication was used to solubilize them. The quality of total RNA was assessed with a TapeStation (Agilent Technologies, Santa Clara, CA, USA), and only samples of high quality (RNA integrity value >8) were included in the analyses. The starting amount of total RNA was $100 \mathrm{ng}$. The labeling and hybridization were done according to the manufacturer's instructions by using Applied Biosystems GeneChipTM WT PLUS Reagent Kit and Manual Target Preparation for GeneChipTM Whole Transcript (WT) Expression Arrays (UserGuide 23 January 2017; Thermo Fisher Scientific). Fifteen micrograms of cRNA were used for single-stranded cDNA-synthesis ( $\operatorname{sscDNA}$ ) and a total of 5,5 ug of sscDNA was fragmented. A total of $2.3 \mu \mathrm{g}$ was hybridized on Clariom S Affymetrix array.

\section{Gene set enrichment and pathway analysis}

Gene set enrichment analysis (GSEA, http://software.broadinstitute.org/gsea/index.jsp)_(13) -was carried out to connect gene expression signatures with previously known gene sets and pathways. The analysis was performed for each cell line and matrix combination separately using the full expression data set against C2: curated gene sets available at broad institute web page. Genes were ranked using signal-to-noise ratio and gene set permutation was used for FDR estimation and enrichment score adjustment. Additional analyses for Gene Ontology enrichment and KEGG pathway visualization were carried out in $\mathrm{R}$ (v. 3.5.3) using packages gage (generally applicable gene set enrichment, v. 2.32.1)_(14) -and pathview (v. 1.22.3)_(15)-. Both the GSEA and additional $R$ analyses were performed by the Functional Genomics Unit (FuGU) at the University of Helsinki. Two samples (UT-SCC-24A/fibrin and UT-SCC-24B/fibrin) were excluded from the analysis as a result of probability of mislabelling.

\section{Analysis of cells circularity and their surface area}

Cells circularity and surface area were measured using ImageJ software (Wayne Rasband, National Institute of Mental Health, Bethesda, MD, USA). The experiments were done four times independently. Two wells were used for each condition and 3 cells were randomly selected and measured from each well. 
All experiments were repeated independently at least three times, each in duplicate or triplicate. Values are given as means \pm standard deviations. All statistical analyses were performed using SPSS (IBM SPSS Statistics for Windows, version 21.0; Armonk NY, IBM Corp.) To determine the statistical significance, we performed one-way analysis of variance (ANOVA) followed by Bonferroni correction. We set statistical significance to $p<0.05$. $P$ values were presented as follows: ${ }^{*}=\mathrm{P} \leq 0.05,{ }^{* *}=\mathrm{P} \leq 0.01,{ }^{* * *}=\mathrm{P} \leq 0.001, * * * *=\mathrm{P} \leq 0.0001$._Origin lab software was used to create the figures.

Results:

243 SCC cells morphology is affected by the matrix type

Cancer cell morphology was affected by the type of matrix (Figure 1). While cells seeded on BSA had similar morphology to the cells in the control wells, cells on Matrigel formed round clusters. Cells on fibronectin had a more flattened surface than cells on the other matrices. Cells on Myogel, fibrin, and collagen were more spindle in shape and there were fibers surrounding the cells. Here we present the pictures of UT-SCC-24B cells only since the other cell lines behaved similarly (data not shown).

For all the cell lines, cells on Matrigel had the tendency of-highest circularity value (above 0.8 out

of 1 ) due to the formation of cell clusters $L_{L}$ though circularity was close to 0.8 in many instances with the other matrices as well (Figure 2). The other matrices did not have any-lear effect on cell eircularity.

The majority of the cell lines, except UT-SCC-24A, seeded on top of fibronectin had higher surface area than in the other conditions, but this difference did not reach statistical significance (Figure 3). UT-SCC-24A cultured on top of fibrin had significantly lower surface area compared to the cells on plastic wells (Figure 3).

\section{Cells-matrix interaction}

260 Scanning electron microscope was used to observe the differences between structures of matrices 261 (Figure 4). While Matrigel has a fiber sheet structure, Myogel's structure was in form of thin 
unorganized fibers together with small globular proteins. Fibrin has abundant thin fibers. Collagen presented helical fibers structure. Fibronectin did not show a fibril structure.

The SEM pictures revealed the interaction between the cells and the matrices (Figure 5). Cells cultured on BSA behaved similarly to the controls. On top of Matrigel, cells formed small clusters. Cells on fibronectin tend to be flat with large surface areas. As for Myogel, cells were gathered in groups and they were in contact with several fibers. For fibrin and collagen, cells were embedded within the matrix fibers.

Fibrin increased and collagen reduced SCC proliferation, while matrix effect on cell migration was cell line dependent.

The proliferation rate for all the tested cell lines was the highest on top of fibrin, and the lowest on top of collagen (Figure 6). This difference was significant for the fibrin-coated wells in case of UTSCC-24A, 24B, and 42A, and for the collagen-coated wells in case of UT-SCC-24A and 42A.

The scratch wound cell migration assay showed that some matrices were able to affect cancer cell migration but this effect was cell line-dependent (Figure 7). Opposite to the proliferation results, collagen_-induced UT-SCC-24B migration and fibrin reduced it. For UT-SCC-42A, cells cultured on top of Myogel were migrating significantly slower compared to the control. Matrigel was not used for migration assay since the cells formed clusters and a homogeneous wound was not possible to be achieved.

\section{Myogel induced SCC cell invasion}

Cancer cells had different invasion speeds in the scratch wound invasion assay based on the matrix used (Figure 8). Cells cultured within Myogel-collagen invaded the fastest. On the other hand, cells did not invade through Myogel-fibrin and fibrin matrices. Myogel was able to induce cancer cells invasion when added to the collagen; significantly in case of UT-SCC-42 A and B. 
292 Figure 10). Myogel was able to significantly

293 induce cancer cell invasion when added to the fibrin in case of UT-SCC-24B and 42A (Figure 10).

304 Fibrin had the highest and BSA and fibronectin the lowest impact on SCC cell molecular profile

In order to understand the mechanism behind the effect of different matrices on the SCC cells behavior, we studied the molecular gene profile using RNA sequencing transcriptome profiles. Matrices were able to change the gene expression of hundreds of genes (Supplementary Table 2). While cells seeded on fibrin had the largest difference (574 and 103 genes significantly affected, $P \leq 0.05$, in UTSCC-24A and B, respectively), cells on BSA (15 and 19 genes significantly affected in UTSCC-24A and B, respectively) and fibronectin ( 9 and 15 genes significantly affected in UTSCC-

312 cells cultured on plastic (Supplementary Table 2). The most significantly affected genes for each 313 matrix are presented in Supplementary Table 3.

314 Gene ontology enrichment analysis revealed several affected ontology groups (Supplementary 315 Table 4). These were both matrix and cell line dependent. The 10 most up- and downregulated 316 biological processes indicated by analysis are presented in Supplementary Table 5. 
Due to the large variation between the two cell lines, we unfortunately were not able to detect specific genes or ontology groups directly responsible for the changes in the SCC cells behavior.

\section{Discussion}

ECMs are increasingly used in cancer research to study different aspects of cancer cell behavior, such as proliferation, migration, invasion and drug testing. The usage of these matrices was regarded as a leap in moving cords-closer to in vivo conditions than the traditional 2D cell culture on plastic. This is mainly due to the ability of these matrices to provide essential elements needed for the cell-cell and cell-matrix interaction. Due to the presence of several types of ECM, 326 such as Matrigel, Myogel, collagen, and fibrin, choosing the most suitable matrix that fits with the needed assays without knowing its properties and effects could be risking the reliability of the results is difficult. Unfortunately, several researchers select the matrix type for their assay based only on the availability, cost, and easiness of the matrix handling, without paying attention to the effects of the matrix on the-cancer cells behavior. Using a non-representative tumor matrix could lead to non-reliable results. In this project, we pointed out the significant differences in SCC cells behavior and their gene profile when tested with various matrices. This emphasizes the importance of selecting the most suitable matrix for each assay.

We first studied the effect of six-five matrices on the HNSCC cell morphology. Interestingly, all the used cell lines formed cell round clusters when cultured on top of Matrigel, which is the most common commercial extracellular matrix used in in vitro experiments. Our results are in line with several other studies showing similar cell behavior on Matrigel in different cancer types_-(16-18). Forming cell clusters may be due to the presence of a large amount of basement membrane proteins in Matrigel which seems to hold the cells together (19). Even though mimicking the basement membrane is considered as an advantage for Matrigel, this feature is a disadvantage in invasion assays due to the difficulties of cancer cells to invade through it. Opposite to the Matrigel, cells cultured on top of Myogel, fibrin and collagen had a spindle shape, which represents more the invasive phenotype of carcinoma cells, as reported in several publications_(16,20,21). This morphology may represent an epithelial-mesenchymal transition (EMT), which is an important feature for cancer cell migration and invasion_(22-24). Cells cultured on fibronectin had a unique flattened shape with a large surface area. This shape could be explained by the presence of the 
$347 \mid \alpha_{5} \beta_{1}$ integrin_(25) which is a fibronectin receptor (26), leading to an interaction that requires 348 traction forces provided by the matrix.

349 To confirm our visual observation of cell morphology, we measured the circularity and surface 350 area of the cells. As expected, cells cultured on top of Matrigel had the highest circularity value 351 due to the formation of round clusters. On the other hand, cells cultured on fibronectin had the 352 highest surface area due to the flat shape of the cells.

353 In order to get a better understanding of the cell-matrix interaction, we visualized the cells and the 354 matrix under scanning electron microscope. As expected, most of the used matrices, except BSA 355 and fibronectin, have fibril structures. The fibril structure of the matrices differed from one matrix 356 to another in the terms of the amount of the fibers (rich vs poor) and thickness of the fibers (thick 357 vs thin). All these differences, in addition to the presence or absence of several growth factors and 358 other proteins, explain the differences in the behavior of cancer cells from one matrix to another. 359 The interaction between the cells and the matrix was also different from one matrix to another. 360 For some matrices, as in Myogel, the cells were surrounded by fibers, while for others, cells were 361 either on top of the matrix (Matrigel) or embedded in it (fibrin and collagen).

362 As cell viability assay is one of the main assays used in in vitro cancer research, we studied if the 363 matrix itself could have an effect on cancer cell proliferation. Interestingly, one pattern was found 364 in all the tested cell lines with the highest proliferation rate detected in the fibrin-coated wells and 365 the lowest in the collagen wells. Our results are in line with Simpson-Haidaris et al. who reported 366 similar results for breast cancer cells MCF-7 cultured on fibrin_(27). On the other hand, our results 367 are opposite to Chen et al., who reported a higher proliferation rate of MCF-7 cells when cultured 368 on a porous collagen scaffold (28), suggesting that the effect of the collagen matrix is cell line 369 dependent. Other matrices did not have a significant effect on HNSCC cell proliferation which goes 370 hand by hand with some studies $(29,30)$.

371 Next, we studied the effect of the different matrices on cancer cell migration. Our results 372 revealed that the effects of the studied matrices on HNSCC migration were cell line dependent, 373 and the significant effects were assured by collagen, Myogel, and fibrin for some cell lines. It 374 was an interest to us to notice the opposite effect of collagen and fibrin matrices on the 375 proliferation and migration behavior of the UT-SCC-24B cell line. While these cells had the 376 highest rate of proliferation on fibrin and the lowest on collagen, the opposite happened in cell 
377 migration. This may return to the fact that the low proliferative cancer cells have high migration 378 capadity and vice versa_(31).

379 Our scratch wound cell invasion assay showed that cells cultured within Myogel-collagen 3D 380 matrix invaded faster than cells within other matrices. This induction of invasion was mainly due 381 to Myogel since we also cultured HNSSC cells within collagen alone and the invasion speed was 382 lower. A similar effect of Myogel was observed on other cell lines (1). Cancer cells did not invade through fibrin or Myogel-fibrin, which might returnmay be due to the fibrin's compact structure. Similar to scratch wound cell invasion assay, Myogel was able to induce invasion in spheroids. However, in scratch wound assay, Myogel-collagen was the most invasive inductive matrix in all 4 cell lines, while in spheroid 3 out of 4 cell lines invaded the fastest in Myogel-fibrin and one in Myogel-collagen. This difference is most likely due to differences in the concentration of the gels in the two assays $(1 \mathrm{mg} / \mathrm{ml}$ in the scratch wound invasion and $0.5 \mathrm{mg} / \mathrm{ml}$ in the spheroid invasion assays). Gels concentration were choosen either following the manufacturer instruction (scratch wound invasion assay) or after lab optimization (spherioid invasion assay). Based on both invasion assays, adding Myogel seems to improve the speed of HNSCC cancer cells invasion. This Myogel 394 Matrigel has been the mostly used matrix for in vitro 3D cancer research. However, it should be 395 kept in mind that it is derived from mouse sarcoma containing mostly basement membrane proteins_(19)-. Due to its nature, in our invasion assays, cells failed to invade efficiently.

Based on our mRNA microarray results, the matrix type was able to significantly affect hundreds of genes and several pathways. Interestingly, these genes and pathways were not shared between matrices or cell lines but were matrix and cell line dependent. This was the reason that 400 401 unfortunately we were not able to detect specific gene or pathway responsible for the changes in the SCC cells behavior. These results indicate that one cell line cannot represent the behavior of any studied tumor type, and always more than one cell line should be used in in vitro experiments.

Our study revealed important effects of the ECMs on HNSCC cells' behavior, morphology, and molecular gene profile. We showed here that the ECMs are not idle elements, but instead, they have significant effects on the in vitro results. We believe that for each assay, selecting the 
407

appropriate matrix, based on its characteristics and the studied cell line, is necessary to get reliable results in in vitro experiments. In theory, selecting human tumor-derived matrix could represent the closest condition to the in vivo tumor microenvironment which increases the reliability of the in vitro cancer cells testing.

\section{Acknowledgment:}

We acknowledge the funders of this study: the Sigrid Jusélius Foundation, The Cancer Society of Finland, Oulu University Hospital MRC grant, the Emil Aaltonen Foundation, Helsinki University Central Hospital Research Funds, and Jane and Aatos Erkkos Foundation.

\section{References:}

(1) Salo T, Dourado MR, Sundquist E, Apu EH, Alahuhta I, Tuomainen K, et al. Organotypic threedimensional assays based on human leiomyoma-derived matrices. Philosophical Transactions of the Royal Society B: Biological Sciences 2018;373(1737):20160482.

(2) Whittaker CA, Bergeron K, Whittle J, Brandhorst BP, Burke RD, Hynes RO. The echinoderm adhesome. Developmental Biology 2006;300(1):252-266.

(3) Provenzano PP, Eliceiri KW, Campbell JM, Inman DR, White JG, Keely PJ. Collagen reorganization at the tumor-stromal interface facilitates local invasion. BMC Medicine 2006;4(1):38.

(4) Sundquist E, Renko O, Salo S, Magga J, Cervigne NK, Nyberg P, et al. Neoplastic extracellular matrix environment promotes cancer invasion in vitro. Experimental cell research 2016;344(2):229-240.

(5) Lu P, Weaver VM, Werb Z. The extracellular matrix: A dynamic niche in cancer progression. Journal of Cell Biology 2012;196(4):395-406.

(6) Quante M, Tu SP, Tomita H, Gonda T, Wang SSW, Takashi S, et al. Bone Marrow-Derived Myofibroblasts Contribute to the Mesenchymal Stem Cell Niche and Promote Tumor Growth. Cancer Cell 2011;19(2):257-272. 
435 (7) Lou Y, Kanninen L, Kuisma T, Niklander J, Noon LA, Burks D, et al. The Use of Nanofibrillar 436 Cellulose Hydrogel As a Flexible Three-Dimensional Model to Culture Human Pluripotent Stem 437 Cells. Stem Cells and Development 2014;23(4):380-392.

438 (8) Salo T, Sutinen M, Hoque Apu E, Sundquist E, Cervigne NK, de Oliveira CE, et al. A novel human 439 leiomyoma tissue derived matrix for cell culture studies. BMC Cancer 2015;15(1):981.

440 (9) Naakka E, Tuomainen K, Wistrand H, Palkama M, Suleymanova I, Al-Samadi A, et al. Fully 441 Human Tumor-based Matrix in Three-dimensional Spheroid Invasion Assay. Journal of Visualized 442 Experiments 2019(147):e59567.

443 (10) Al-Samadi A, Poor B, Tuomainen K, Liu V, Hyytiäinen A, Suleymanova I, et al. In vitro 444 humanized 3D microfluidic chip for testing personalized immunotherapeutics for head and neck 445 cancer patients. Experimental Cell Research 2019;383(2):111508.

446 (11) Almahmoudi R, Salem A, Murshid S, Dourado RM, Apu HE, Salo T, et al. Interleukin-17F Has 447 Anti-Tumor Effects in Oral Tongue Cancer. Cancers 2019;11(5).

448 (12) Tuomainen K, Al-Samadi A, Potdar S, Turunen L, Turunen M, Karhemo P, et al. Human Tumor449 Derived Matrix Improves the Predictability of Head and Neck Cancer Drug Testing. Cancers $450 \quad 2019 ; 12(1)$.

451 (13) Subramanian A, Tamayo P, Mootha VK, Mukherjee S, Ebert BL, Gillette MA, et al. Gene set 452 enrichment analysis: A knowledge-based approach for interpreting genome-wide expression 453 profiles. Proceedings of the National Academy of Sciences USA 2005;102(43):15545.

454 (14) Luo W, Friedman MS, Shedden K, Hankenson KD, Woolf PJ. GAGE: generally applicable gene 455 set enrichment for pathway analysis. BMC Bioinformatics 2009;10(1):161.

456 (15) Luo W, Brouwer C. Pathview: an R/Bioconductor package for pathway-based data integration 457 and visualization. Bioinformatics 2013;29(14):1830-1831.

458 (16) Truong D, Puleo J, Llave A, Mouneimne G, Kamm RD, Nikkhah M. Breast Cancer Cell Invasion 459 into a Three Dimensional Tumor-Stroma Microenvironment. Scientific Reports 2016;6(1):34094. 460 (17) Beers J, Gulbranson DR, George N, Siniscalchi LI, Jones J, Thomson JA, et al. Passaging and 461 colony expansion of human pluripotent stem cells by enzyme-free dissociation in chemically 462 defined culture conditions. Nature Protocols 2012;7(11):2029-2040. 
463 (18) Polo ML, Arnoni MV, Riggio M, Wargon V, Lanari C, Novaro V. Responsiveness to PI3K and 464 MEK Inhibitors in Breast Cancer. Use of a 3D Culture System to Study Pathways Related to 465 Hormone Independence in Mice. PLOS ONE 2010;5(5):e10786.

466 (19) Zhang Y, Lukacova V, Reindl K, Balaz S. Quantitative characterization of binding of small 467 molecules to extracellular matrix. The Journal of Biochemical and Biophysical Methods 468 2006;67(2):107-122.

469 (20) Chen Y, Lan H, Wu Y, Yang W, Chiou A, Yang M. Epithelial-mesenchymal transition softens 470 head and neck cancer cells to facilitate migration in 3D environments. Journal of Cellular and 471 Molecular Medicine 2018;22(8):3837-3846.

472 (21) Hakkinen KM, Harunaga JS, Doyle AD, Yamada KM. Direct Comparisons of the Morphology, 473 Migration, Cell Adhesions, and Actin Cytoskeleton of Fibroblasts in Four Different Three474 Dimensional Extracellular Matrices. Tissue Engineering Part A 2011;17(5-6):713-724.

475 (22) Son H, Moon A. Epithelial-mesenchymal Transition and Cell Invasion. Toxicological research 476 2010;26(4):245-252.

477 (23) Kalluri R, Weinberg RA. The basics of epithelial-mesenchymal transition. Journal of Clinical 478 Investigation 2009;119(6):1420-1428.

479 (24) Zhou P, Li B, Liu F, Zhang M, Wang Q, Liu Y, et al. The epithelial to mesenchymal transition 480 (EMT) and cancer stem cells: implication for treatment resistance in pancreatic cancer. Molecular 481 Cancer 2017;16(1):52.

482 (25) Ahmedah HT, Patterson LH, Shnyder SD, Sheldrake HM. RGD-Binding Integrins in Head and 483 Neck Cancers. Cancers 2017;9(6):56.

484 (26) Wang K, Seo BR, Fischbach C, Gourdon D. Fibronectin Mechanobiology Regulates 485 Tumorigenesis. Cellular and molecular bioengineering 2016;9:1-11.

486 (27) Simpson-Haidaris P, Rybarczyk B. Tumors and Fibrinogen. Annals of the New York Academy of 487 Sciences 2001;936(1):406-425.

488 (28) Chen L, Xiao Z, Meng Y, Zhao Y, Han J, Su G, et al. The enhancement of cancer stem cell 489 properties of MCF-7 cells in 3D collagen scaffolds for modeling of cancer and anti-cancer drugs. 490 Biomaterials 2012;33(5):1437-1444. 
491 (29) Hurst RE, Kyker KD, Bonner RB, Bowditch RD, Hemstreet,George P.,,3rd. Matrix-dependent 492 plasticity of the malignant phenotype of bladder cancer cells. Anticancer Research 2003 493 Jul;23(4):3119-3128.

494 (30) Fliedner FP, Hansen AE, Jørgensen JT, Kjær A. The use of matrigel has no influence on tumor 495 development or PET imaging in FaDu human head and neck cancer xenografts. BMC Medical 496 Imaging 2016;16(1):5.

497 (31) Mouneimne G, Brugge JS. YB-1 Translational Control of Epithelial-Mesenchyme Transition. 498 Cancer Cell 2009;15(5):357-359.

Figure legends:

Figure 1. SCC cell morphology observed under light microscope. Different shapes of UT-SCC-24B cells were observed depending on the used matrix. Cells on BSA gave similar morphology to the control. Cells were clustered on Matrigel, flat on fibronectin, and spindle on Myogel, fibrin, and collagen. Scale bar $=100 \mu \mathrm{m}$

Figure 2. SCC cell circularity. UT-SCC cells were cultured on different matrices and plastic (control) for 3 days and pictured on day 3 under light microscope. Cell circularity was measured using ImageJ software. In all cell lines represented in the figure, cells cultured on top of Matrigel 509 showed the highest circularity value (above 0.8). Data are presented as means \pm standard deviations. ${ }^{*} \mathrm{P} \leq 0.05 . \mathrm{N}=3$.

Figure 3. SCC cell surface area. UT-SCC cells were cultured on different matrices and plastic 512 (control) for 3 days and pictured on day 3 under light microscope. Cell surface area was measured 513 using ImageJ software. UT-SCC-24B, UT-SCC-42A and UT-SCC-42B cells showed the highest cell 514 surface area when cultured on Fibronectin, but this difference did not reach statistical significance. 515 Data are presented as means \pm standard deviations. ${ }^{*} \mathrm{P} \leq 0.05 . \mathrm{N}=3$.

Figure 4. Matrices structure observed under scanning electron microscope. Coverslips were coated with different matrices and prepared for scanning electron microscope. Matrigel has a 519 fiber sheet structure. Myogel's structure was in form of thin unorganized fibers together with 
small globular proteins. As for fibrin, its fibers were thin. Collagen presented helical fibers structure. BSA and fibronectin did not show a fibril structure. Scale bar $=1 \mu \mathrm{m}$

Figure 5. SCC cells and matrix interaction observed under scanning electron microscope. UT-SCC $42 \mathrm{~B}$ cells were cultured on coated coverslips with different matrices and prepared for scanning electron microscope. Cells cultured on BSA coated wells did not have any interaction with the matrix, similarly to the cells cultured on plastic. For Matrigel, cells formed small clusters on top of the matrix. Cells on fibronectin tend to be flat, more than any studied matrix, with a large surface area. As for Myogel, cells were gathered in groups and they were in contact with several fibers. In fibrin and collagen, cells were embedded within the matrix fibers. Scale bar $=10 \mu \mathrm{m}$

Figure 6. SCC cell proliferation rate on different matrices. UT-SCC cells were cultured on different matrices for three days and the cell proliferation rate was measured using luminescent cell viability assay. The proliferation rate for all the cell lines was the highest on fibrin and the lowest on collagen. This difference was significant for the fibrin-coated wells in case of UT-SCC-24A, 24B, and $42 \mathrm{~A}$ cell lines and also for collagen in case of UT-SCC-24A and $42 \mathrm{~A}$ cell lines. The red line represents the control value. Data are presented as means \pm standard deviations. ${ }^{*} \mathrm{P} \leq 0.05,{ }^{* *} \leq$ $0.01, * * * \leq 0.001, * * * * \leq 0.0001 . \mathrm{N}=3$

Figure 7. SCC cell migration on different matrices. UT-SCC cells were cultured on different matrices and cell migration was evaluated using scratch wound cell migration assay. The migration rate was dependent on both the matrix and the cell line. Data are presented as migration curves and area under the curves as means \pm standard deviations. ${ }^{*} \mathrm{P} \leq 0.05,{ }^{* *} \leq 0.01, * * * \leq 0.001, * * * *$ $\leq 0.0001$. N=3.

Figure 8. SCC cells invasion through different matrices. UT-SCC cells were cultured on different matrices and cell invasion was evaluated using scratch wound cell invasion assay. The four studied cell lines showed the fastest invasion rate when cultured on Myogel-collagen and they did not invade through fibrin and Myogel-fibrin. Data are presented as invasion curves and area under the curves as means \pm standard deviations. ${ }^{*} \mathrm{P} \leq 0.05,{ }^{* *} \leq 0.01,{ }_{-} * * * \leq 0.001,{ }^{* * * *} \leq 0.0001 . \mathrm{N}=3$.

Figure 9. Spheroid invasion observed under light microscope. UT-SCC 42A cells were cultured in ultra-low attachment 96-well round bottom plate wells and embedded in different matrices. Spheroids were observed under light microscope. Scale bar $=100 \mu \mathrm{m}$.

Figure 10. SCC spheroid invasion though different matrices. UT-SCC cells were cultured in ultralow attachment 96-well round bottom plate wells and embedded in different matrices. For UTSCC-24A, 42A, and 42B, Myogel-fibrin matrix showed the fastest spheroids invasion, followed by Myogel-collagen. For UT-SCC-24B cells invaded faster in Myogel-collagen followed by Myogelfibrin. Data are presented as invasion curves and area under the curves as means \pm standard 
564 List of Supporting Information:

569 Supplementary Table 1. HNSCC cell lines details. Clinical and pathological characteristics of the $570 \quad$ HNSCC cell lines. TNM is based on

571 Supplementary Table 2. Number of differentially expressed genes of UT-SCC-24A and B cultured 572 on different matrices. Results of mRNA microarray showing the number of differentially expressed 573 genes between cells cultured on plastic and cells cultured on matrices. The genes that passed the 574 filter criteria had a $p<0.05$ and a fold change $\leq-2$ or $\geq 2$. Transcriptome analysis console software 575 was used to analyze the data

576 Supplementary Table 3. The most affected genes of UT-SCC cells cultured on different matrices. 577 Results of mRNA microarray showing the most significantly affected genes (up- down-regulated) 
578 by each matrix we used. The genes that passed the filter criteria had a $p<0.05$ and a fold change $\leq-$

5792 or $\geq 2$. Transcriptome analysis console software was used to analyze the data.

580 Supplementary Table 4. Number of differentially expressed pathways of UT-SCC-24A and B 581 cultured on different matrices. Results of the gene set enrichment analysis (GSEA) showing the 582 number of the differentially represented pathways between cells cultured on plastic and cells 583 cultured on matrices. The pathways that passed the filter criteria had a $p<0.05$.

584 Supplementary Table 5. The $\mathbf{1 0}$ most affected pathways of UT-SCC cells cultured on different 585 matrices. Results of the gene set enrichment analysis (GSEA) showing the 10 most differentially 586 expressed pathways between cells cultured on plastic and cells cultured on matrices. The 587 pathways that passed the filter criteria had a $\mathrm{p}<0.05$.

588

589 Supplementary video 1: migration of the UT-SCC-42A cells on top of Myogel.

590 Supplementary video 2: the invasion of the UT-SCC-42A within Myogel-collagen.

591

592

593

594 


\section{Control}

BSA

Matrigel

Fibronectin

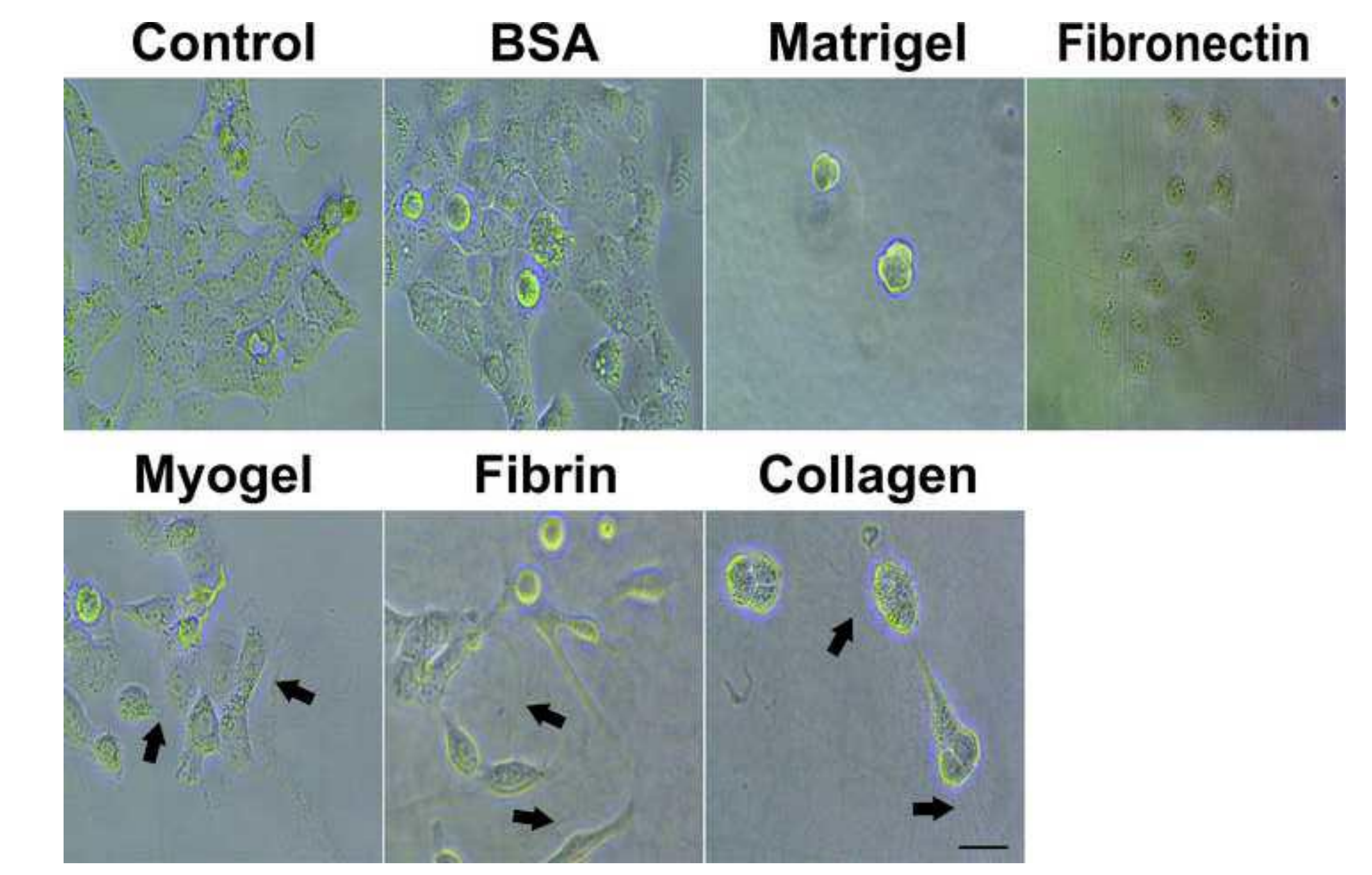

\section{Fibronectin}
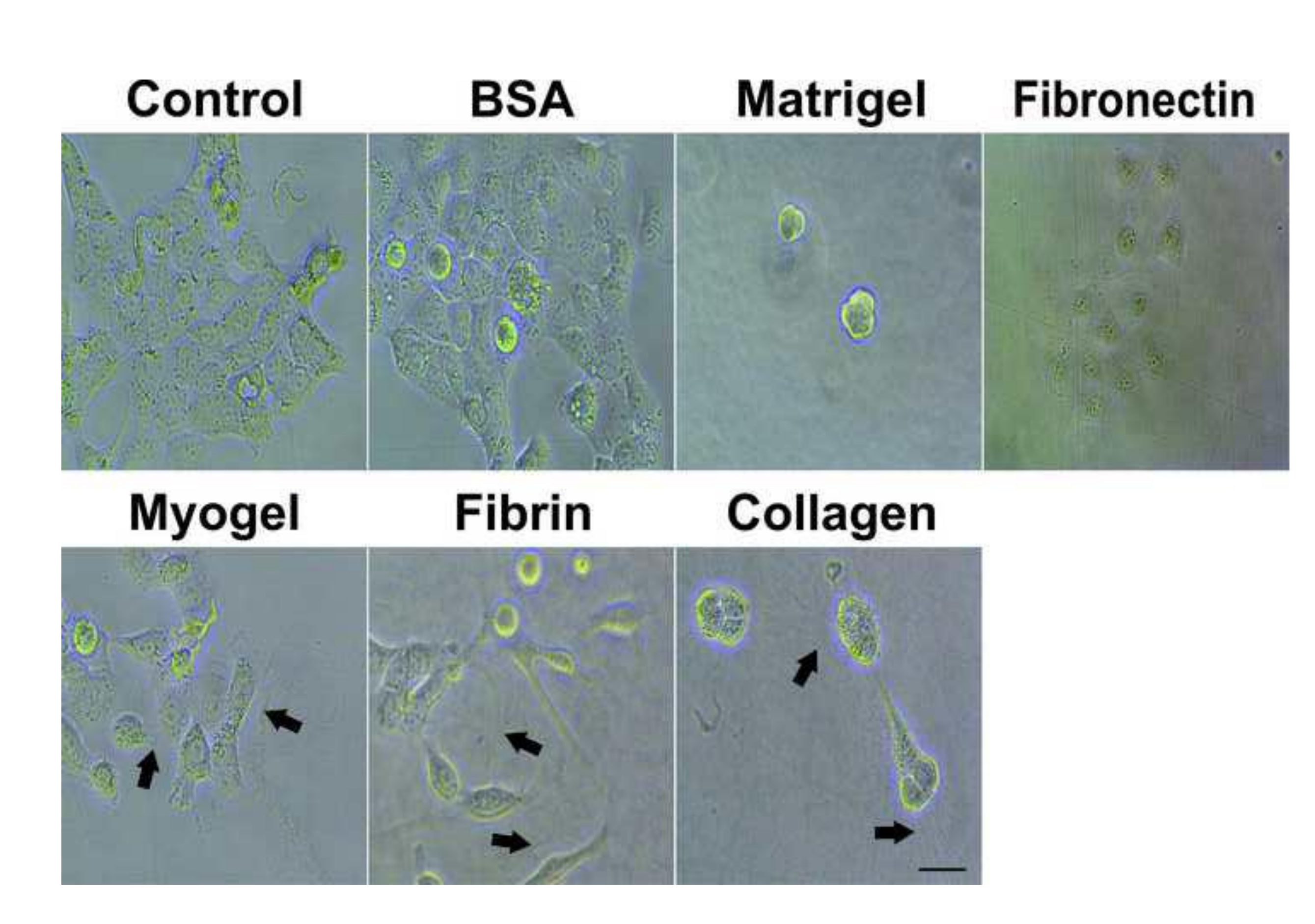

.
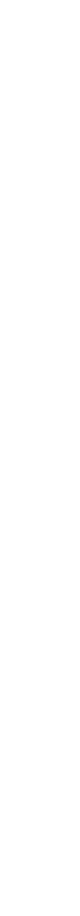
UT-SCC-24A

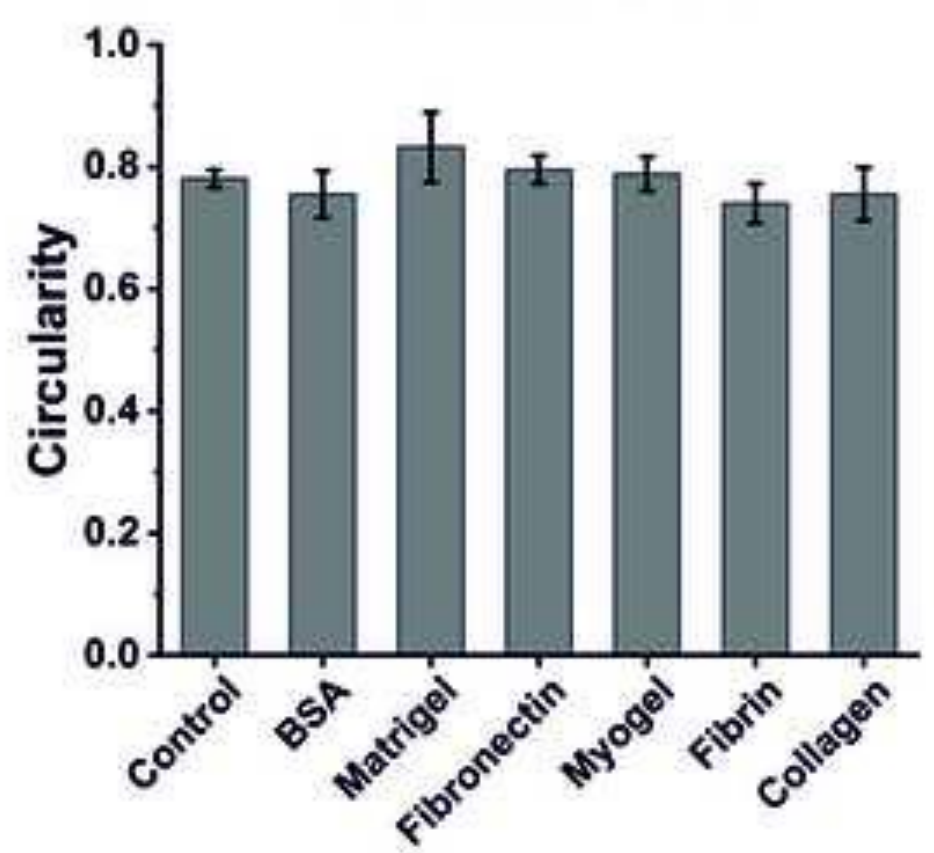

UT-SCC-42A

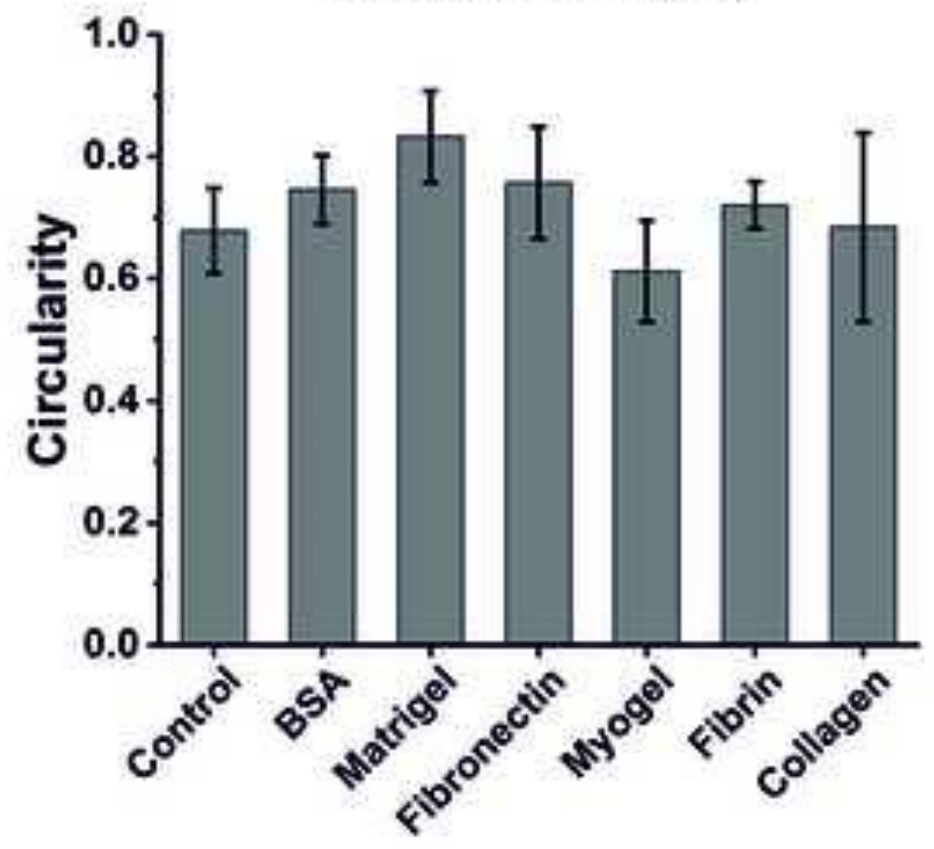

UT-SCC-24B

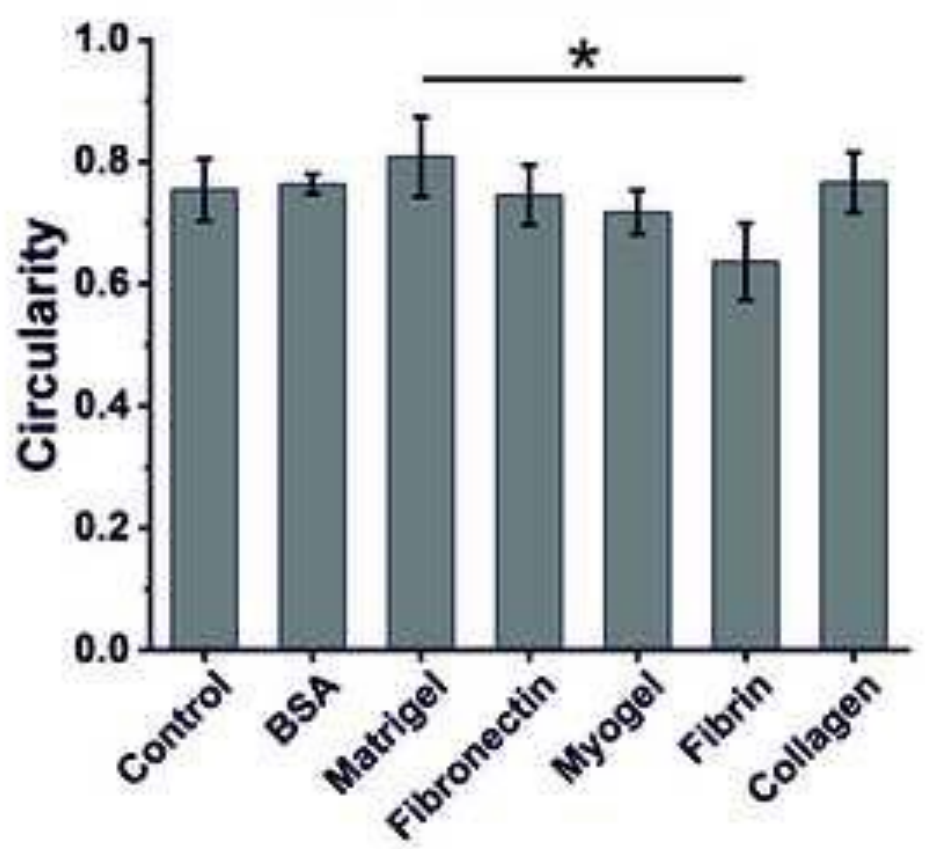

UT-SCC-42B

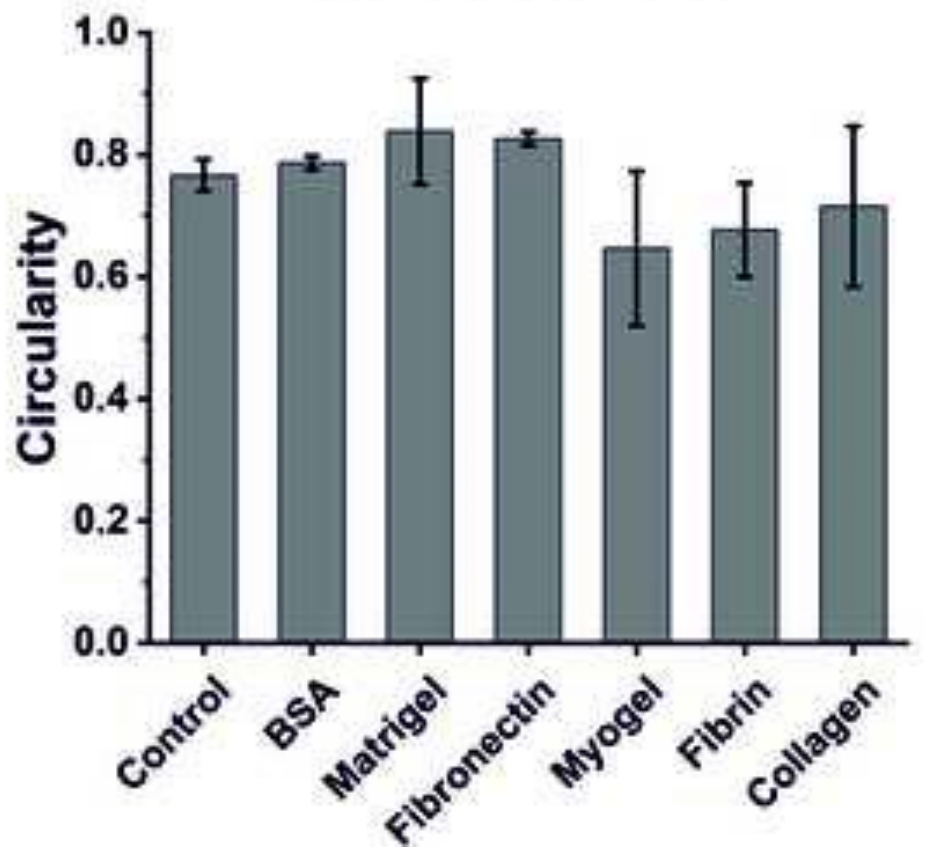



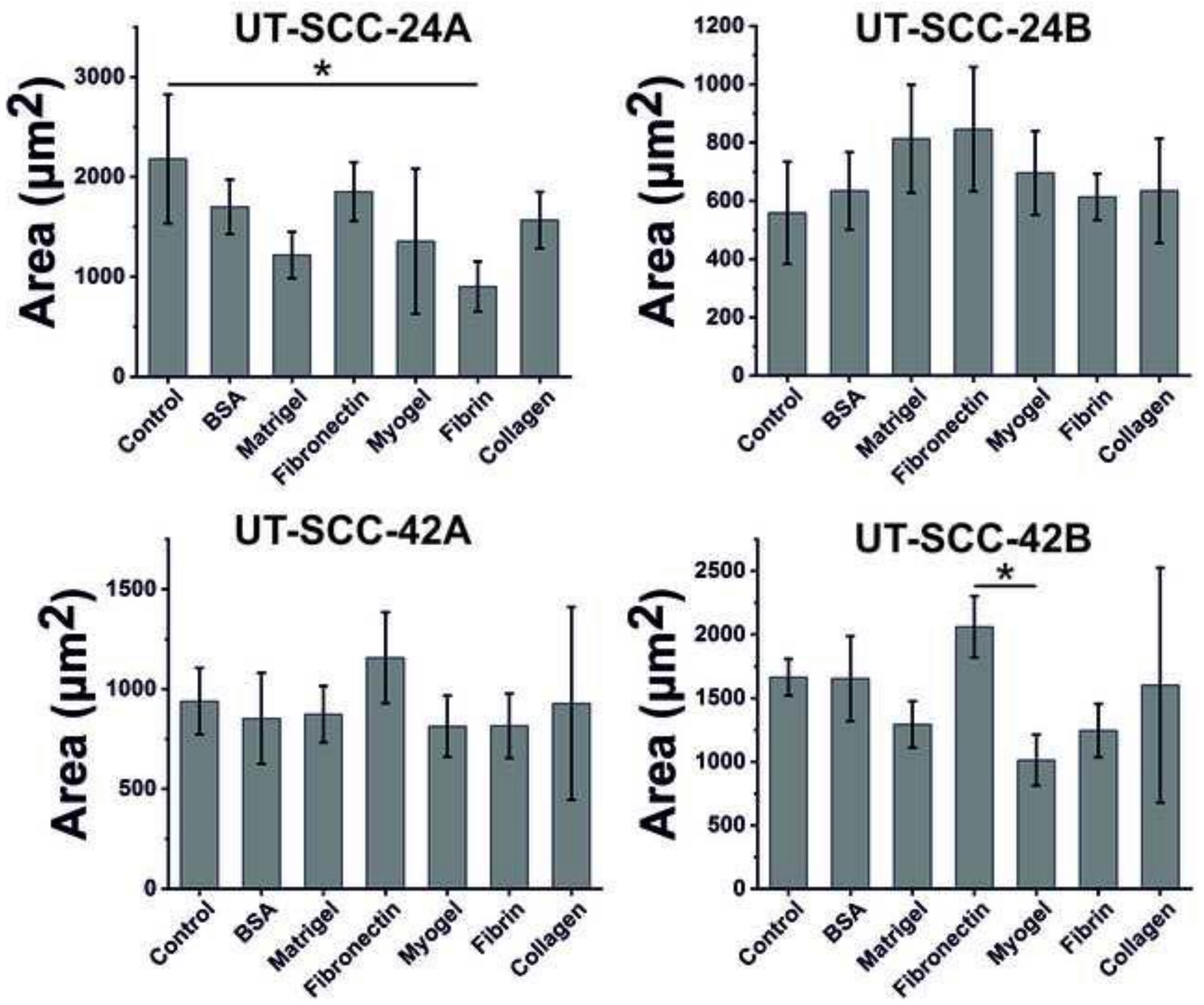
Fibronectin
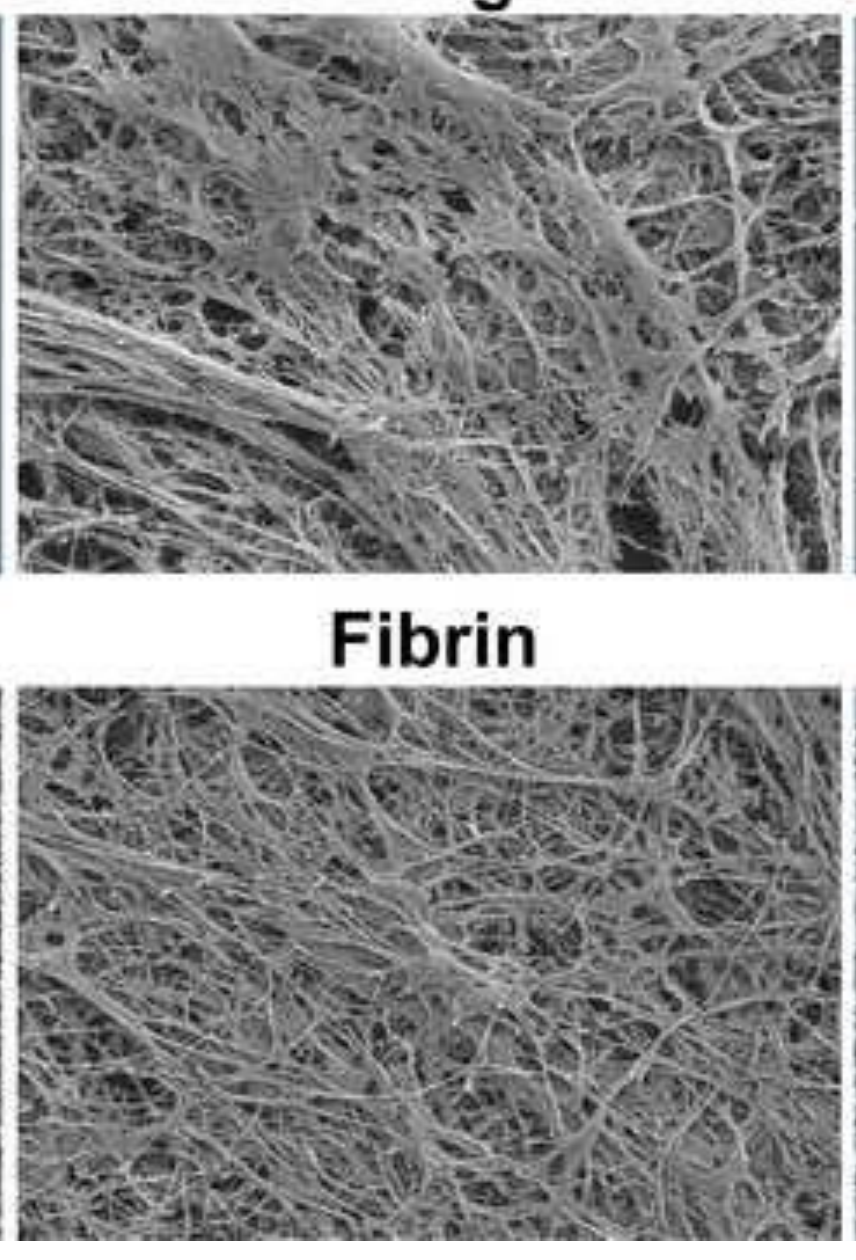

\section{Collagen}
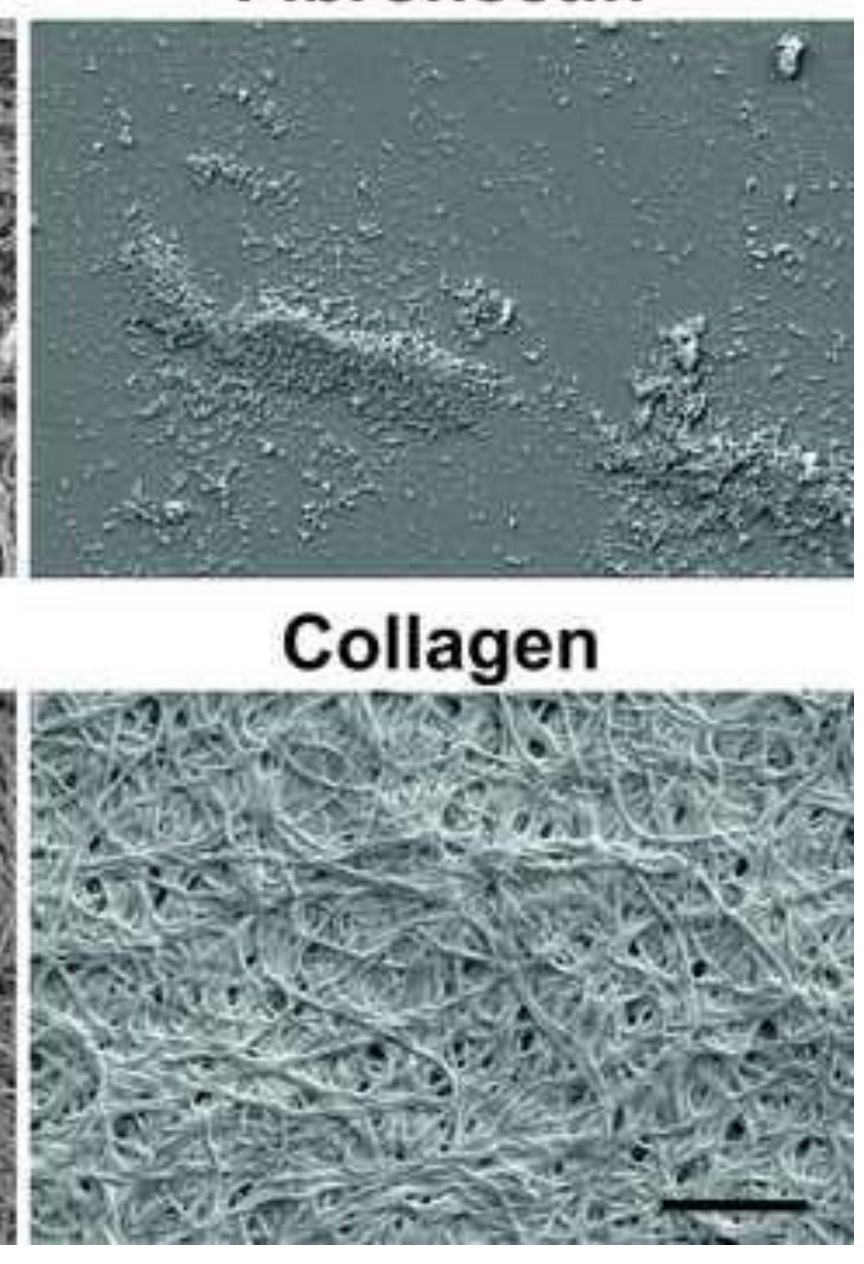

\section{BSA}

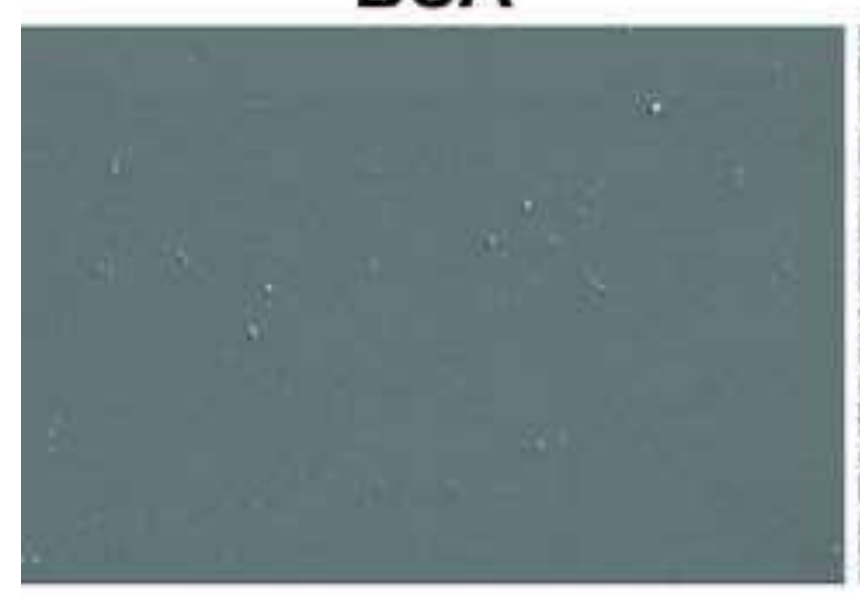

Myogel

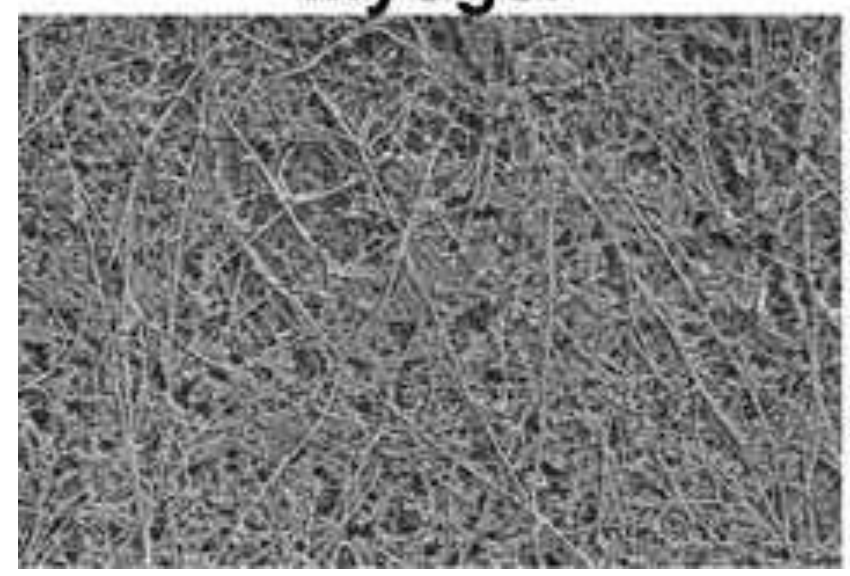

Fibrin

Matrigel . 


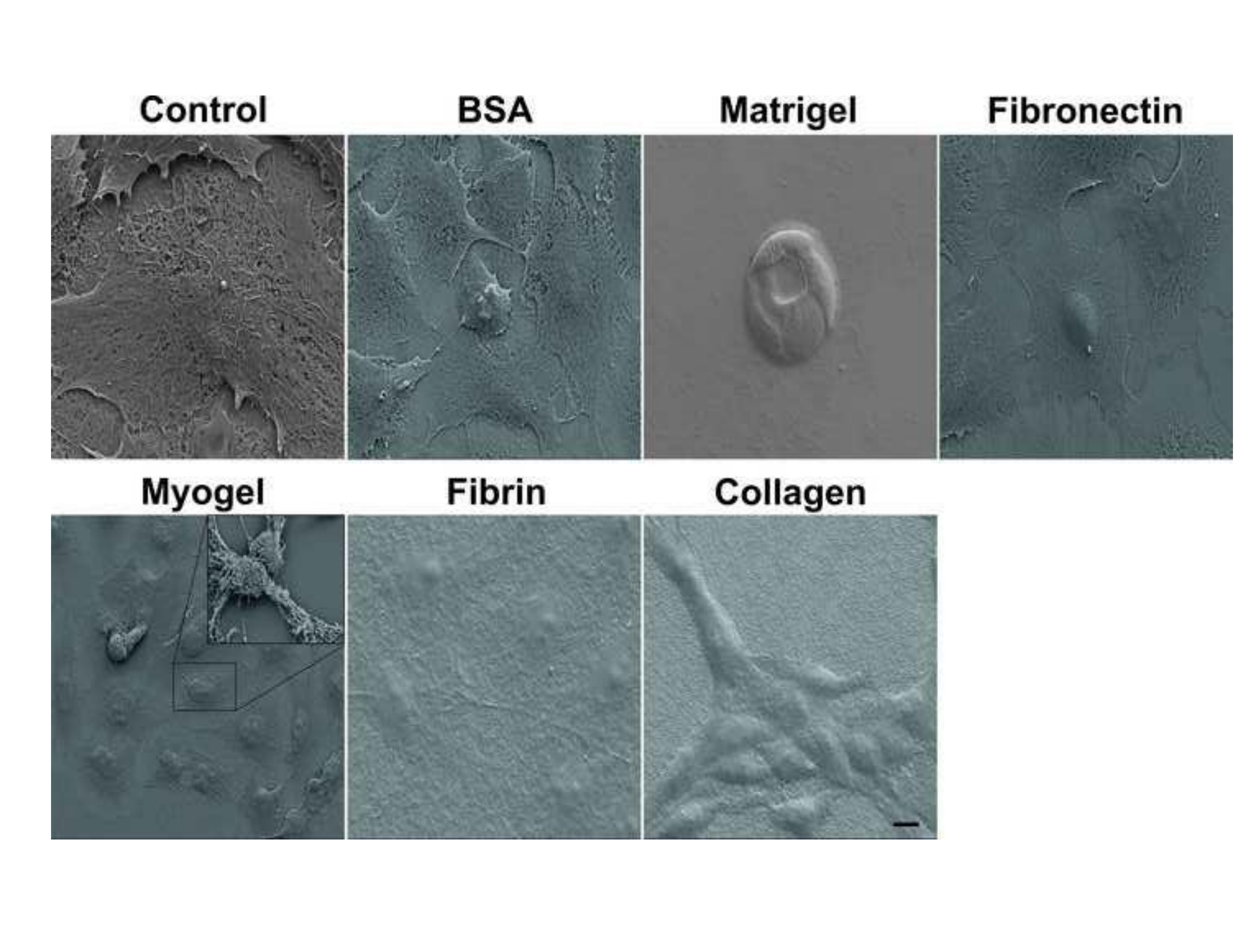

Fibronectin

Myogel

Fibrin

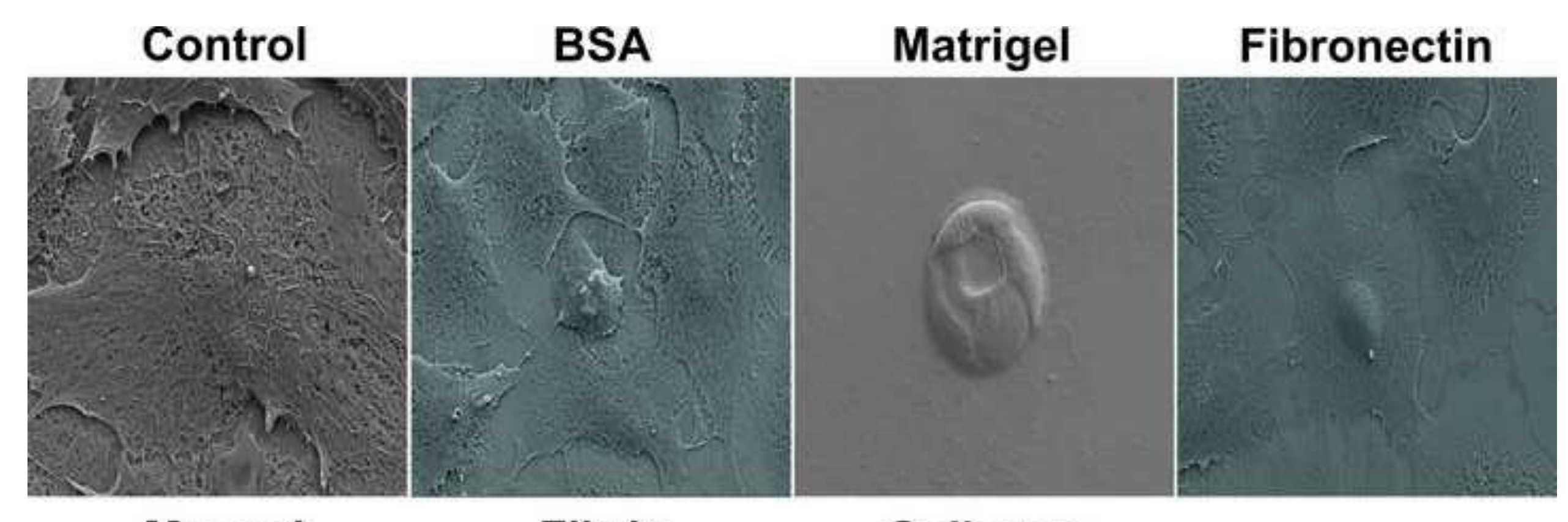

BSA

atrigel
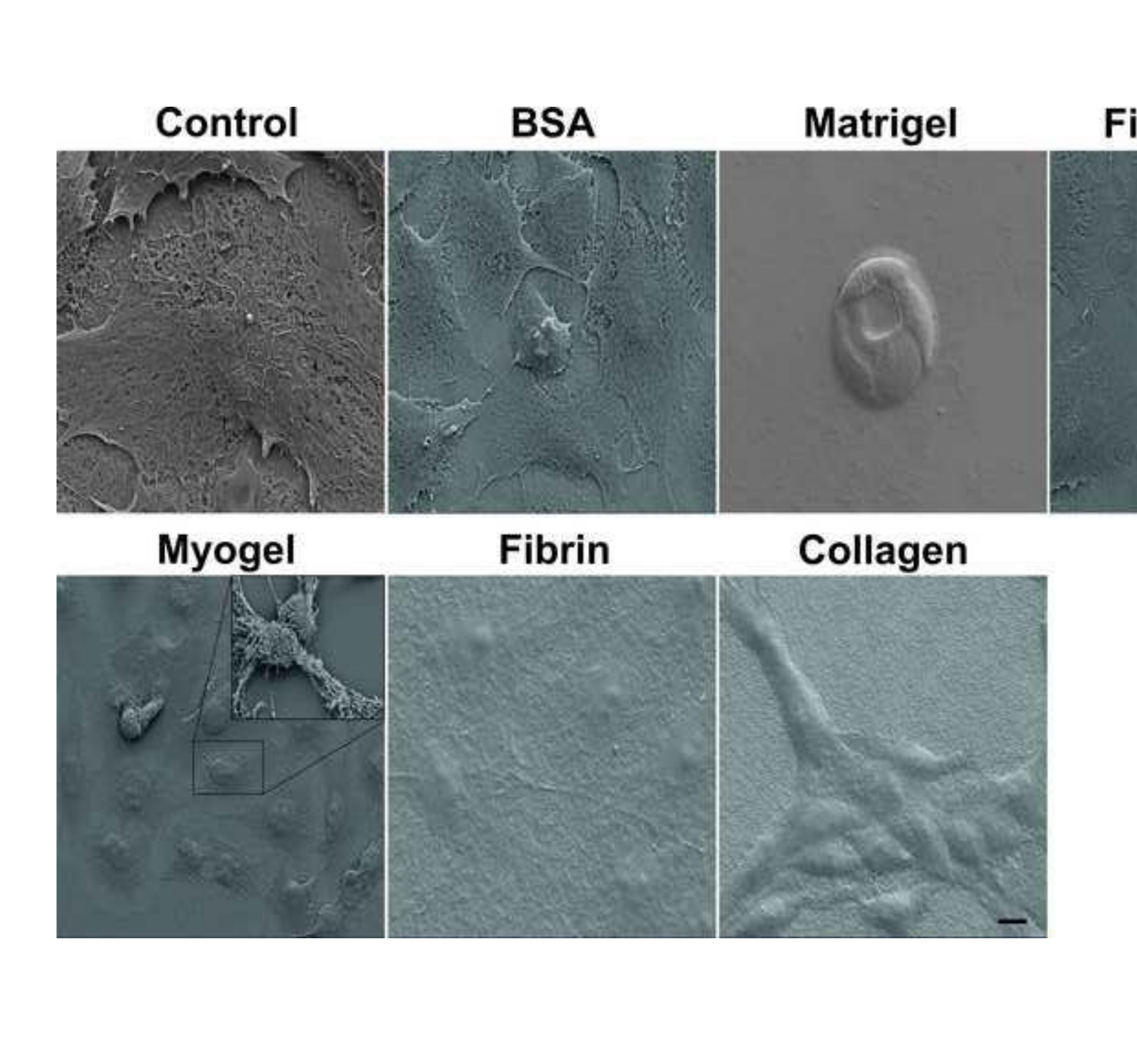

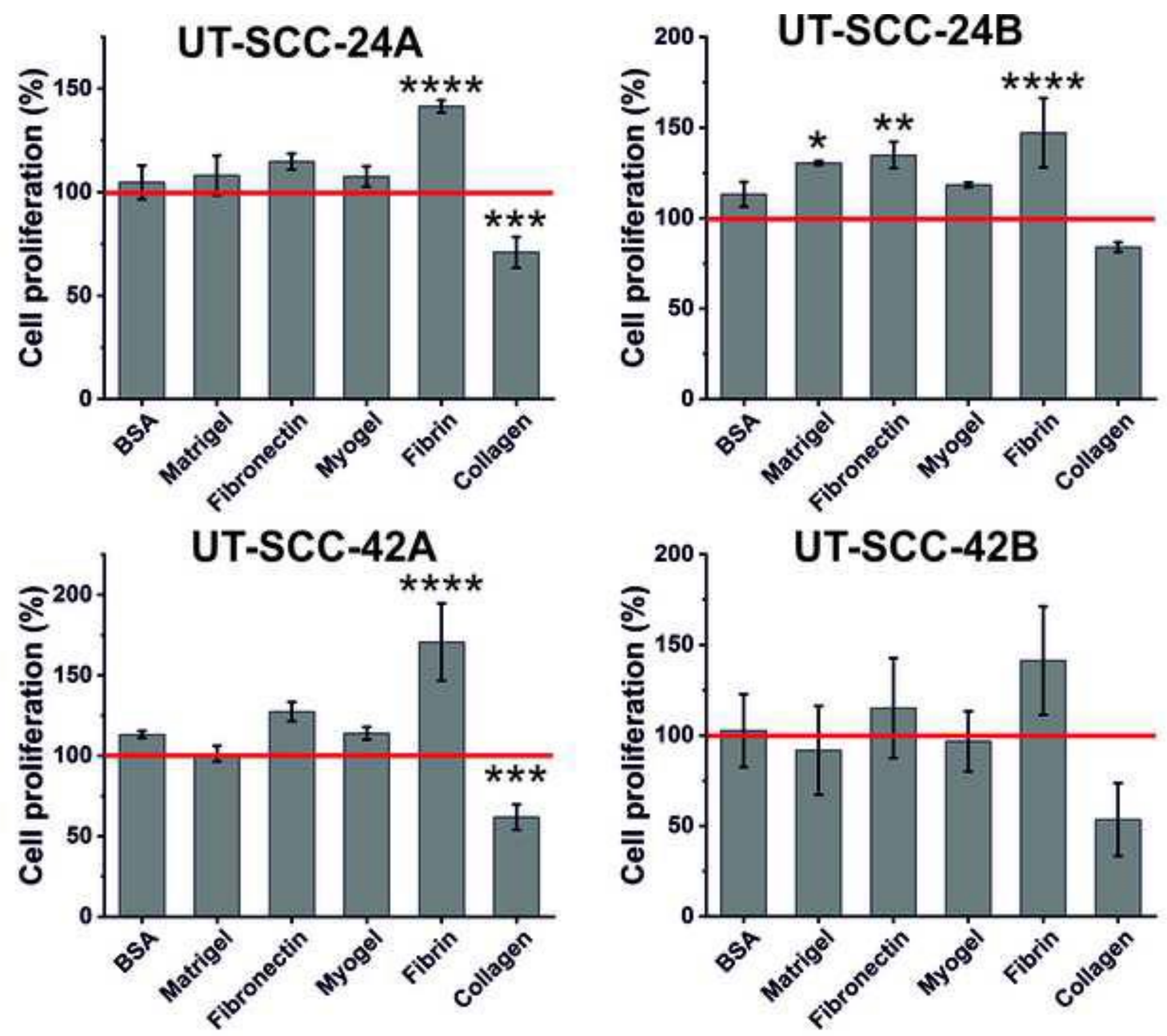
$\rightarrow$-Control- - BSA - Fibronectin $\rightarrow$ Myogel-Fibrin -4 Collagen
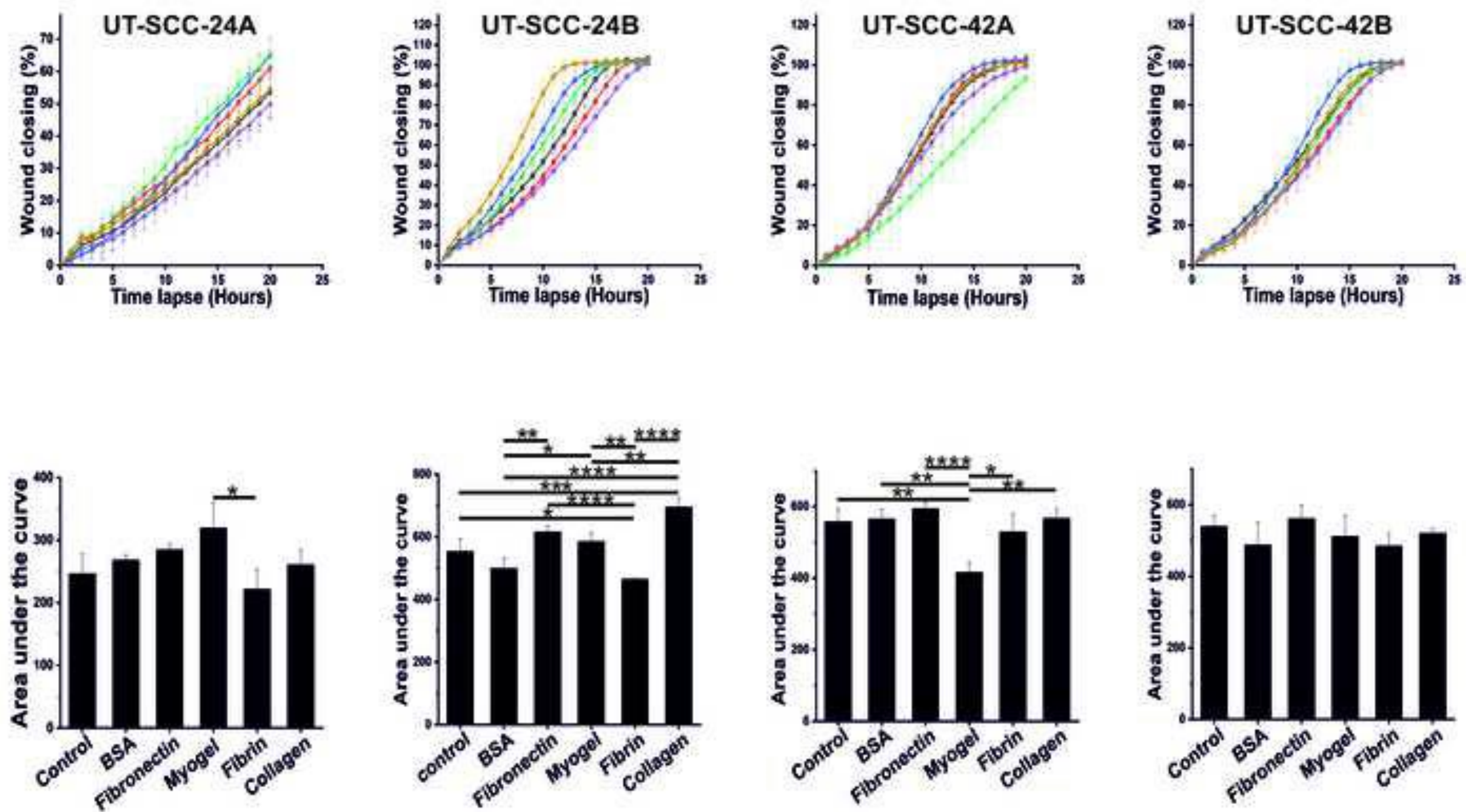
$\rightarrow$-Collagen - -Myogel-Collagen - Fibrin $-\nabla$ Myogel-Fibrin
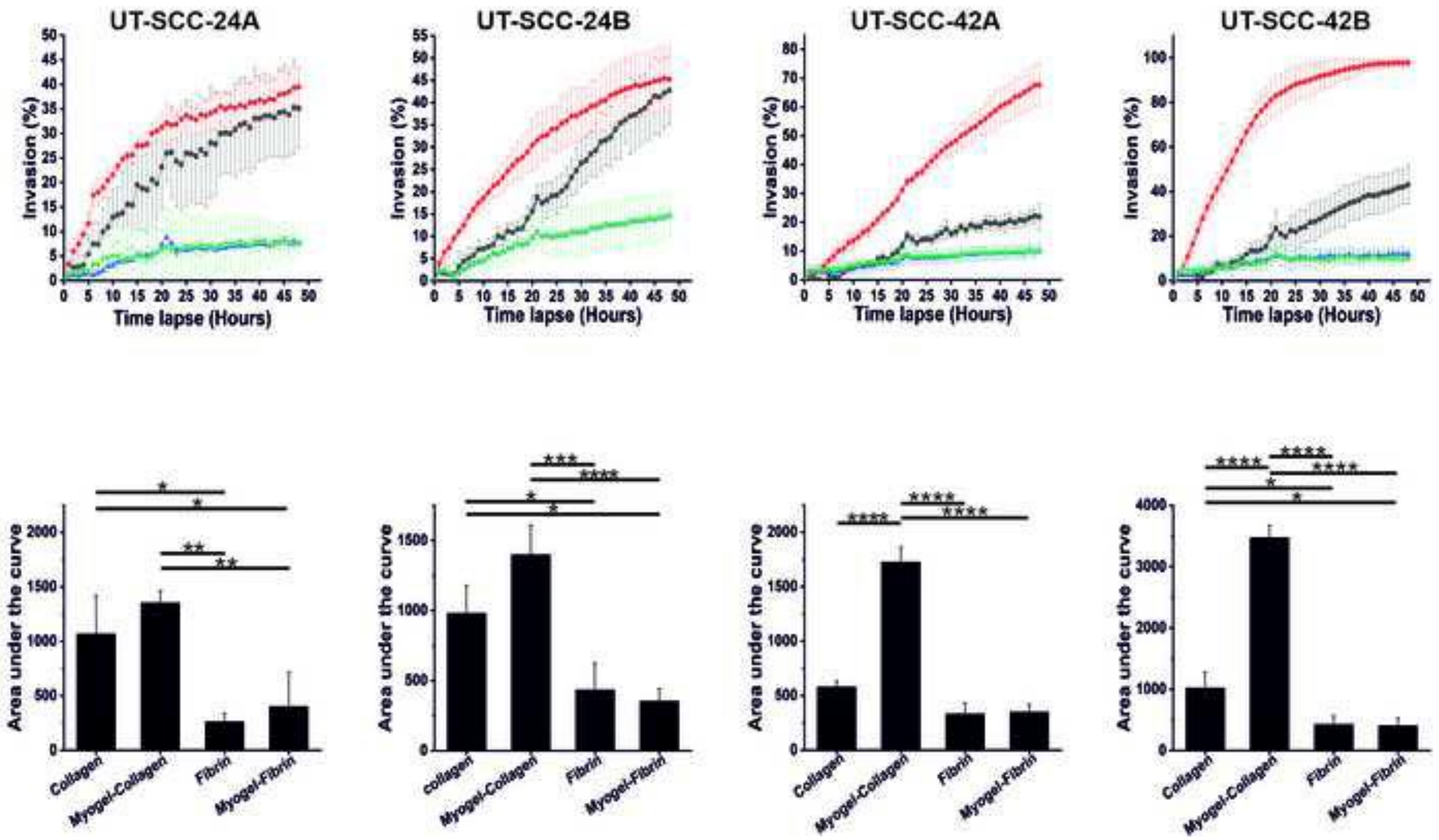
Day 0

Control

Collagen

Myogel-
Collagen

Fibrin

Matrigel
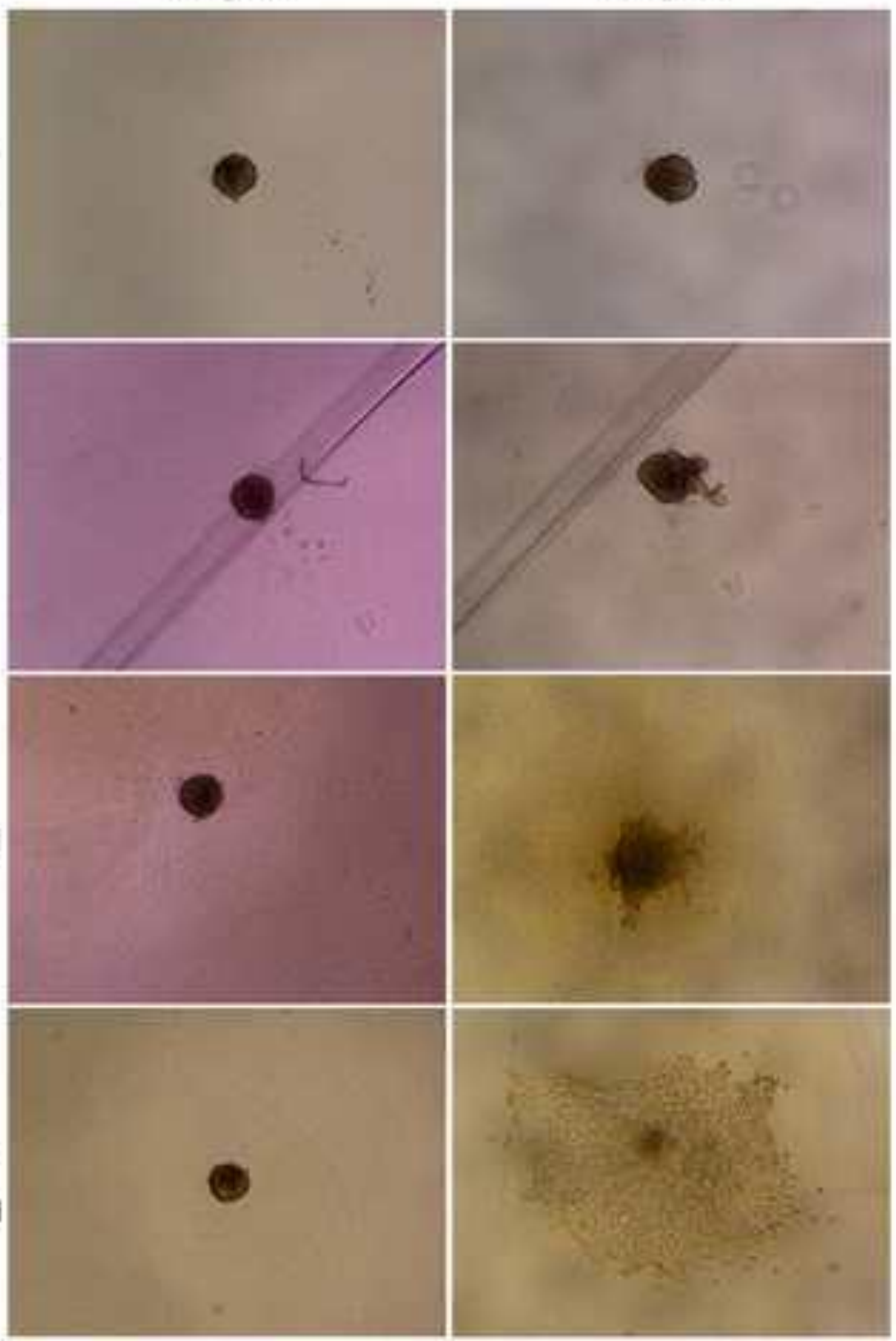

Day 3
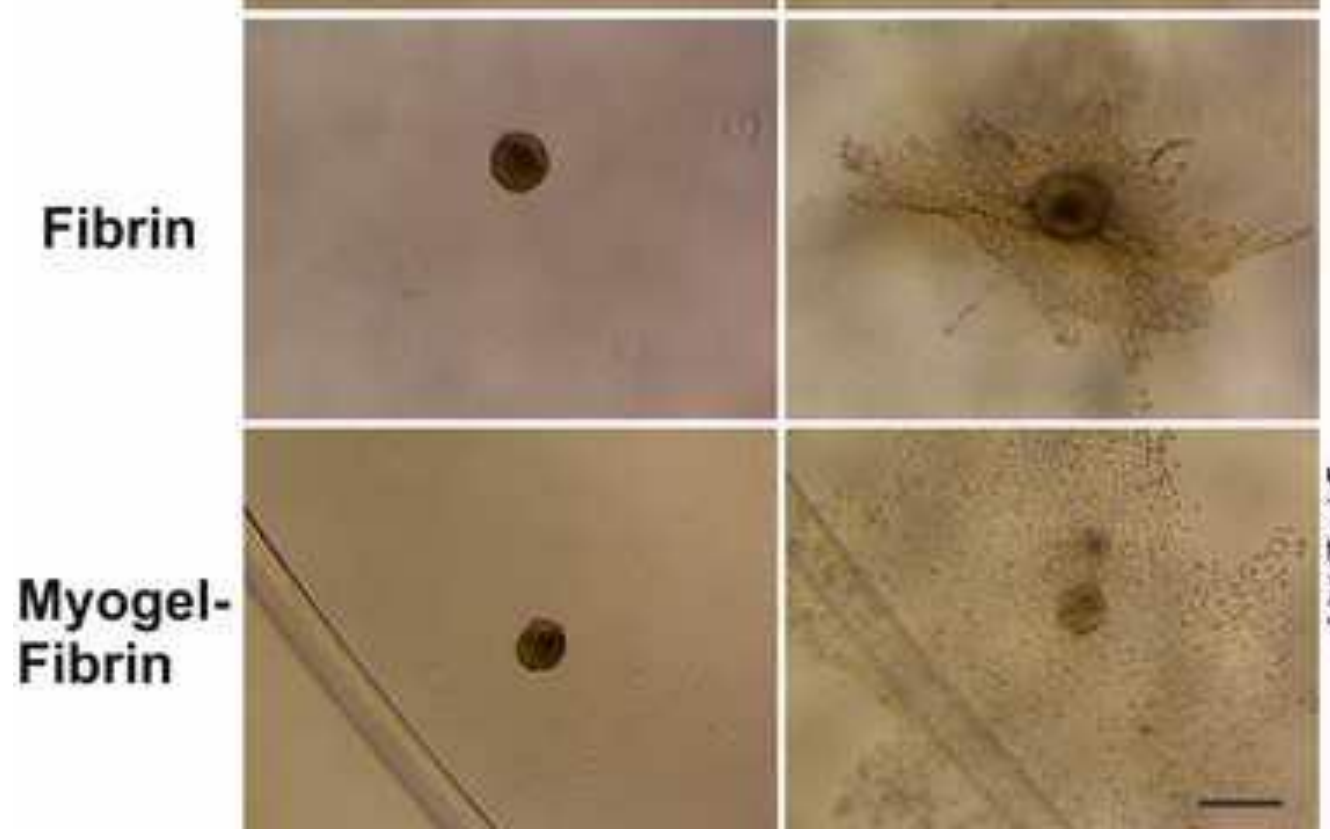

Day 3
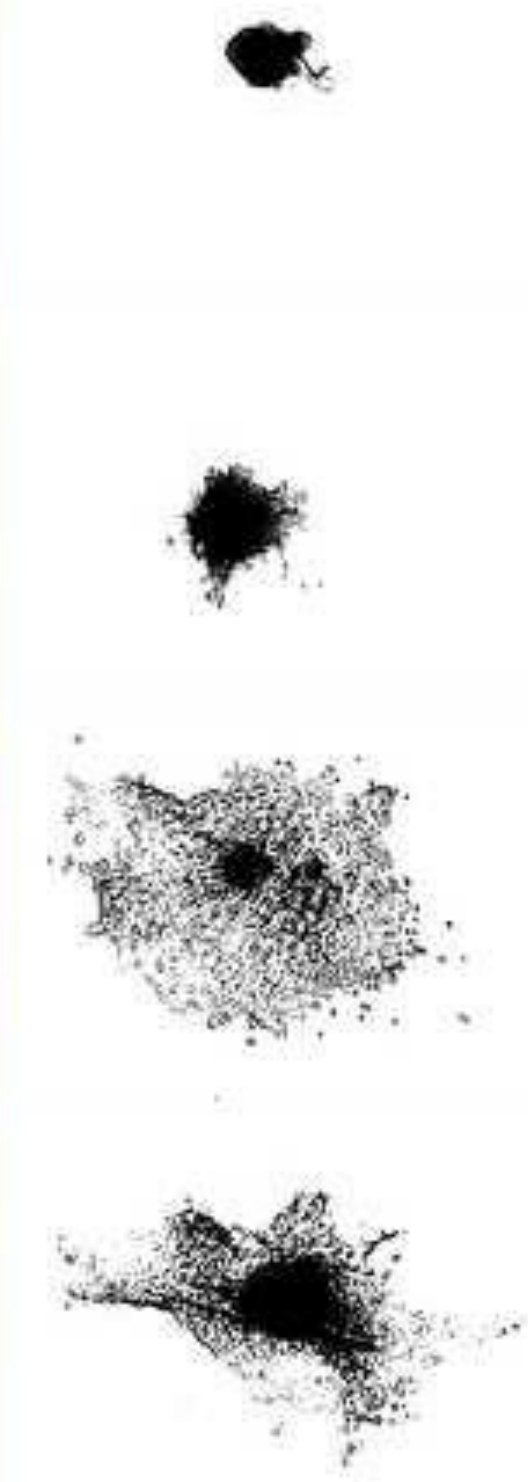

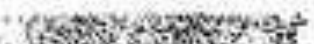

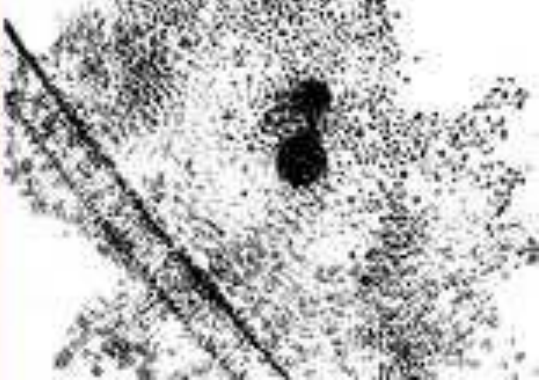


$\rightarrow$ Control-C-Matrigel $\_$Collagen $\longrightarrow$ Myogel-Collagen $-\leftarrow$ Fibrin - Myogel-Fibrin
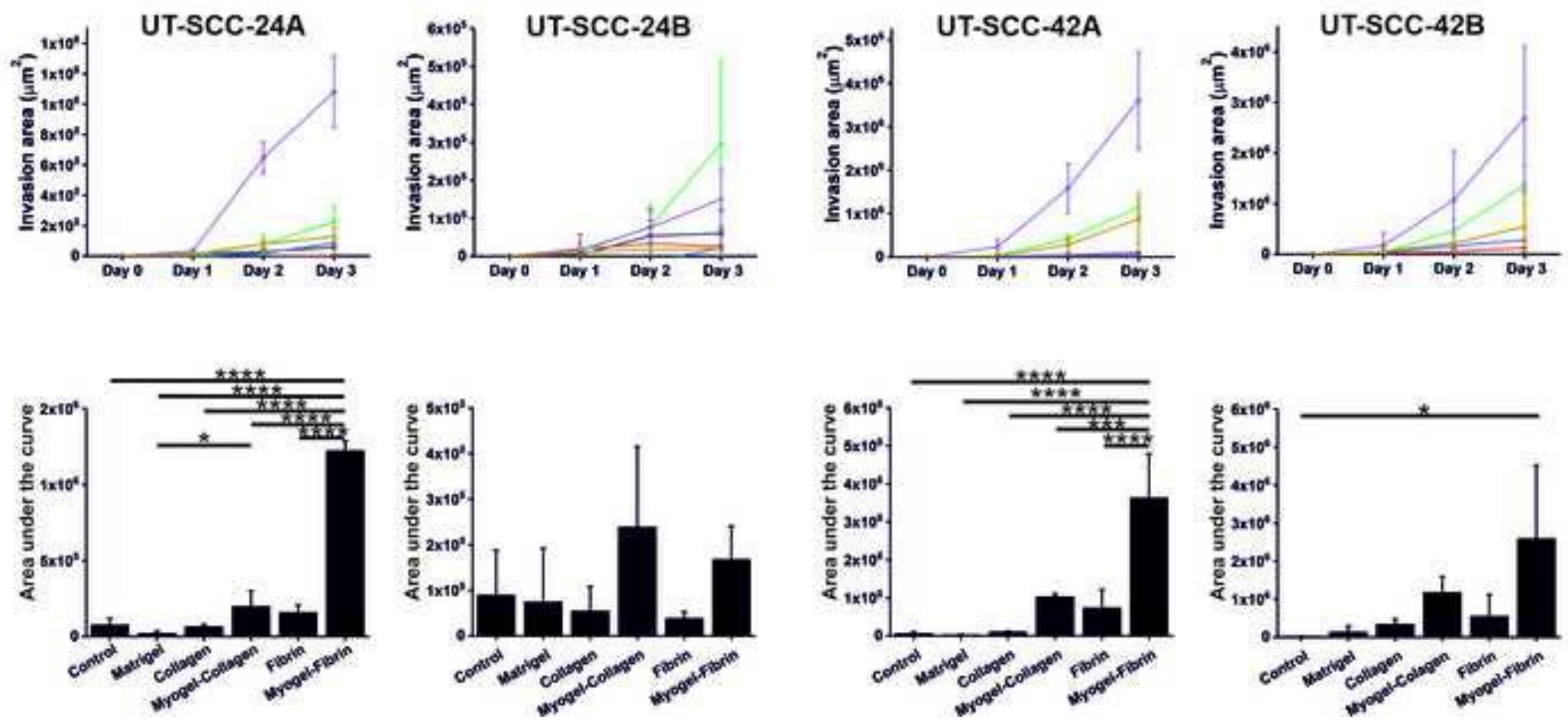
Click here to download Supplementary material for online publication only: Supplement Video 1.mp4 
Video 2
Click here to download Supplementary material for online publication only: Supplement Video $2 . \mathrm{mp} 4$

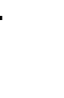


upplementary material for online publication only

Click here to download Supplementary material for online publication only: Table 1.docx 

Click here to download Supplementary material for online publication only: Table 2.docx

\begin{abstract}
(a)
\end{abstract}

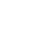

(1)

$\sqrt{3}$

$\sqrt{3}$

$\sqrt{3}$

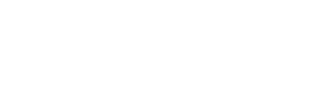
(1) 

Click here to download Supplementary material for online publication only: Table 3.docx

(a)

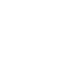

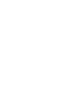

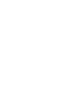

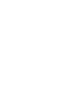

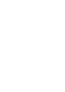

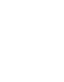
更

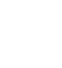

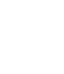

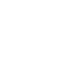

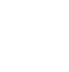

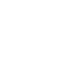

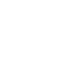

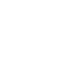

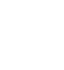
更

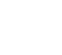



Click here to download Supplementary material for online publication only: Table 4.docx

\begin{abstract}
.
\end{abstract}
(1) . (1) (1) (1) (1) (1) . . . . . 

Click here to download Supplementary material for online publication only: Table 5.docx

\begin{abstract}
(a)
\end{abstract}

(1)

(1)

$\sqrt{2}$

(1)

$\sqrt{10}$

(1)

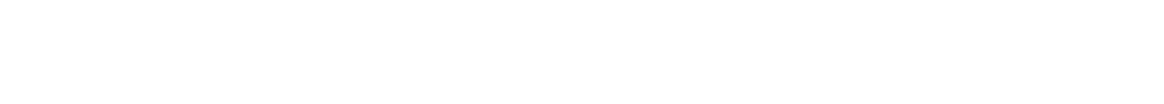

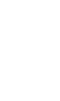

$\sqrt{20}$

$\sqrt{20}$

$\sqrt{20}$

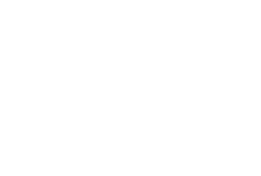

. 


\section{Authors' contributions:}

Wafa Wahbi: Conceptualization, Methodology, Validation, Formal analysis, Software, Investigation , Data Curation, Writing - Original Draft , Writing - Review \& Editing , Visualization, Project administration.

Ahmed Al-Samadi: Conceptualization, Methodology, Validation, Formal analysis, Software, Investigation, Data Curation, Writing - Review \& Editing, Visualization, Project administration, Supervision.

Tuula Salo: Conceptualization, Methodology, Supervision, Writing - Review \& Editing.

Reidar Grenman: Conceptualization, Methodology, Writing - Review \& Editing.

Erika Naakka: Conceptualization, Data Curation, Formal analysis, Writing - Review \& Editing

Katja Tuomainen: Conceptualization, Data Curation, Formal analysis, Writing - Review \& Editing.

Ilida Suleymanova: Software, Writing - Review \& Editing.

Annamari Arpalahti: Data Curation, Writing - Review \& Editing.

Ilkka Miinalainen: Data Curation, Writing - Review \& Editing.

Juho Vaananen: Formal analysis, Writing - Review \& Editing.

Outi Monni: Methodology, Writing - Review \& Editing. 


\section{Declaration of interests}

$\bigotimes$ The authors declare that they have no known competing financial interests or personal relationships that could have appeared to influence the work reported in this paper.

$\square$ The authors declare the following financial interests/personal relationships which may be considered as potential competing interests:

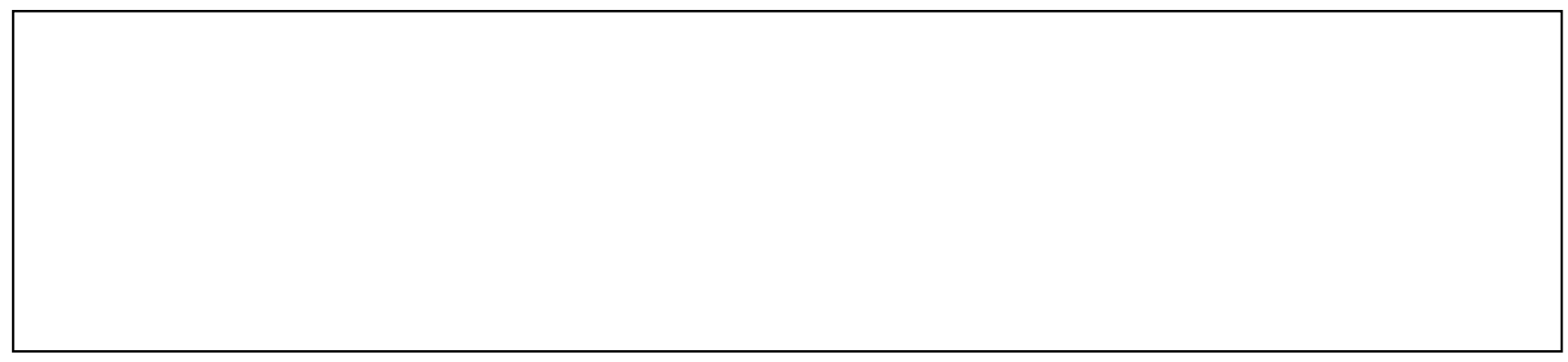

